\title{
Suspensions of prolate spheroids in Stokes flow. Part 1. Dynamics of a finite number of particles in an unbounded fluid
}

\author{
By IVAN L. CLAEYS $\uparrow$ AND JOHN F. BRADY \\ Division of Chemistry and Chemical Engineering, California Institute of Technology, Pasadena, \\ CA 91125 , USA
}

(Received 15 August 1991 and in revised form 18 December 1992)

\begin{abstract}
A new simulation method is presented for low-Reynolds-number flow problems involving elongated particles in an unbounded fluid. The technique extends the principles of Stokesian dynamics, a multipole moment expansion method, to ellipsoidal particle shapes. The methodology is applied to prolate spheroids in particular, and shown to be efficient and accurate by comparison with other numerical methods for Stokes flow. The importance of hydrodynamic interactions is illustrated by examples on sedimenting spheroids and particles in a simple shear flow.
\end{abstract}

\section{Introduction}

The motion of small particles in viscous fluids is relevant to fields as disparate as chemical engineering, biology, soil mechanics, geophysics, materials science and the microelectronics industry (Happel \& Brenner 1973; Kim \& Lawrence 1988). It pertains to the locomotion of micro-organisms, the transport of blood cells in arteries, and the diffusional approach of substrates to enzymes. More traditionally, low-Reynoldsnumber hydrodynamics have been used to analyse the processing of slurries, colloids and composites. If some of the particles are fixed, the results apply to petroleum recovery in porous rocks, or to the monitoring and control of particulate contaminants in cleanroom environments. Suspension rheology is also at the core of active efforts in constitutive equation modelling for polymeric and liquid crystalline solutions.

Much is known at present about the motion of single, rigid particles in creeping flow. This knowledge is directly applicable to the analysis of dilute suspensions, in which each particle is effectively isolated. Unfortunately, this approach breaks down at extremely low concentrations for dispersions of slender bodies, because anisometric solid inclusions interact hydrodynamically much more strongly than spheres do at the same volume fraction. Semi-dilute fibre suspensions for example display extremely high extensional viscosities (Batchelor 1971; Shaqfeh \& Fredrickson 1990; Mewis \& Metzner 1974). This tremendous increase in the resistance of fluids to stretch by the addition of a minuscule amount of fibrous material is of great technological importance, and is one of the factors that prompted us to study ovary particle shapes rather than planetary.

The behaviour of concentrated dispersions remains a challenge for rheologists. For spherical particles, dynamic microstructural simulations have provided valuable insight into the flow behaviour of suspensions up to very high particle densities (Brady \& Bossis 1988; Phung \& Brady 1992). Similar attempts for dispersions of elongated

† Present address: Solvay Research \& Technology, rue de Ransbeek 310, 1120 Brussels, Belgium. 
particles, however, have thus far neglected hydrodynamic interactions, despite their importance, and modelled the suspension as a gas of impenetrable needles, incorporating solvent effects in an ad hoc manner (Bitsanis, Davis \& Tirell 1988, 1990). The numerical difficulties experienced with continuum descriptions of fibre suspensions in the semi-dilute concentration regime and near solid boundaries also reflect the importance of viscous interactions and the difficulty in treating them adequately (Rosenberg, Denn \& Keunings 1990). The need exists, clearly, for a hydrodynamically rigorous simulation method for dispersions of elongated particles. In addition, few numerical or theoretical investigations are available to date in the literature on the rheology of suspensions of thick rods, with moderate aspect ratios. Theories for fibre dispersions usually employ a slowly converging expansion in inverse powers of the logarithm of the aspect ratio, and their predictive value is thus limited to extremely slender bodies. This is unfortunate since most particles and macromolecules of practical importance have eccentricities intermediate between spheres and very slender rods (which tend to either break or bend (Salinas \& Pittman 1981)).

We present herein a new simulation method for suspensions of prolate spheroids of arbitrary aspect ratio, which accurately accounts for hydrodynamic interactions, including many-body effects. It is similar in concept to the successful simulation technique for dispersions of spheres known as Stokesian dynamics (Brady \& Bossis 1988). Only systems consisting of a finite number of particles are considered here first in order to demonstrate the essence of the new approach and its advantages. These simple systems also serve to highlight the effect of viscous interactions, which are hard to distill from simulations of infinite suspensions. Two-body dynamics are also interesting in their own right. For instance, they determine the tendency of freely suspended rods flowing through highly porous fixed beds to line up in the streamwise direction (Shaqfeh \& Koch 1988). Companion papers discuss the extension of our method to unbounded suspensions and illustrate its effectiveness with selected results on the transport properties of uniformly aligned and equilibrium hard-ellipsoid structures (Claeys \& Brady 1993a,b). The model suspension consists of rigid nonBrownian prolate spheroids interacting hydrodynamically in a Newtonian, incompressible fluid at zero Reynolds number (based on the largest particle dimension). Since the body is axisymmetric, this is the simplest geometry retaining the essential novel feature of interest: orientability. It encompasses the sphere at one end of the spectrum and slender round-ended rods at the other.

As the inertia of particles up to several tenths of a millimetre in size can often be neglected relative to viscous retardation in common liquids, the equations governing fluid motion in suspension rheology are usually taken to be the Stokes equations:

$$
\begin{gathered}
\nabla p=\mu \nabla^{2} u, \\
\nabla \cdot u=0 .
\end{gathered}
$$

( $\mu$ represents the viscosity of the suspending fluid; $\boldsymbol{u}$ is the velocity field, and $p$ the associated pressure.) Even though the creeping flow equations $(1.1 a, b)$ are linear, very few analytical solutions are known which satisfy the no-slip boundary condition on two or more immersed particle surfaces. Many numerical techniques for Stokes flow problems have therefore been developed (Weinbaum, Ganatos \& Yan 1990). We shall focus on the most efficient approaches for suspension rheology, which solve the governing equations $(1.1 a, b)$ for the particulate dynamics without determining the entire flow field.

The first class of methods is based on a discretization of the boundary integral equation (Ladyzhenskaya 1963, chap. 3), which writes the velocity at any point $x$ of the 
(bounded) flow domain in terms of the force density and fluid motion at the boundary a $V$ :

$$
u(x)=\frac{-1}{8 \pi \mu} \int_{i V} J(x-y) \cdot \sigma(y) \cdot \mathrm{d} A_{y}+\frac{3}{4 \pi} \int_{i V} \frac{(x-y)(x-y)(x-y)}{|x-y|^{5}}: u(y) \mathrm{d} A_{y}
$$

with

$$
\boldsymbol{\sigma}=-\boldsymbol{p} \boldsymbol{\delta}+\mu\left(\boldsymbol{\nabla} \boldsymbol{u}+{ }^{\mathrm{T}} \boldsymbol{\nabla} \boldsymbol{u}\right) .
$$

The stress tensor $\sigma$ is evaluated at $\boldsymbol{y}$ on the boundary. $\mathrm{d} \boldsymbol{A}_{y}$ is an elementary surface element of $\partial V$, pointing into the control volume $V$; the subscript $y$ indicates that the integration is carried out with respect to the vector $y$. The first integral therefore represents the disturbance produced by a surface distribution of point forces with strength $\boldsymbol{\sigma} \cdot \mathrm{d} \boldsymbol{A}_{\boldsymbol{y}}$. Their effect propagates with the Oseen tensor

$$
J(r)=\frac{\delta}{|\boldsymbol{r}|}+\frac{\boldsymbol{r r}}{|\boldsymbol{r}|^{3}}
$$

A straightforward application of the divergence theorem shows that the second term on the right-hand side of (1.2) vanishes for rigid particles for any $\boldsymbol{x}$ in the flow domain.

In essence, boundary element methods evaluate (1.2) at $\partial V$, apply the appropriate boundary conditions and solve the resulting Fredholm integral equation by discretizing the surface. Coincidentally, the first article exposing a numerical application of this technique considered Stokes flow past spheroids (Youngren \& Acrivos 1975). As many as 64 surface patches were needed to determine the torque exerted by simple shear flow on the particles to an accuracy of $1 \%$ or better. Although the programming aspects have become more refined since, this work illustrates the high computational cost of the technique. More effective numerical approaches, collectively known as multipole techniques, are now available for a wide class of creeping flow problems, restricting the use of the boundary element method to systems with deformable interfaces or complicated geometries. Its newest variant, the completed double-layer solution (Kim, Fuentes \& Karilla 1991), promises much greater efficiency for this type of problems, but will probably not compete with multipole techniques for the simulation of dispersions of particles of relatively simple shape. Incidentally, the multipole expansion solution presented in this work may guide the selection of the appropriate range completer for the completed double-layer method, and thereby increase the performance of the technique for many-body problems involving spheroidal particles.

The multipole collocation method has mostly been applied to the motion of two or three spheres (Hassonjee, Ganatos \& Pfeffer 1988) and to axisymmetric flows past chains of ellipsoids (Gluckman, Pfeffer \& Weinbaum 1971; Liao \& Krueger 1980). It is best suited for problems involving a finite number of identical particles positioned in a very symmetric arrangement. Basically, the strengths of stress singularities located at the centres of the particles are determined by simultaneously satisfying the no-slip condition on all spheroids on a number of judiciously chosen rings on their surface. Since all unknown coefficients are found collectively as the solution of one matrix equation, many-body effects are incorporated. Surface-averaged properties such as the drag can be calculated quite accurately using this technique. However, as the particle separation decreases, many terms must be retained in Lamb's fundamental solution (Lamb 1932), on which the method is based. Also, as recognized by Hassonjee et al. (1988), the computer memory and computation time requirements are rather large, and compare unfavourably with the needs of Stokesian dynamics (see §2). Although the 'multipoles' were described merely as 'multilobular disturbances' originally (Gluckman et al. 1971), Weinbaum et al. (1990) have shown for spheres that they correspond to moments of the stress density on the particle surface, i.e. to the Stokeslet, 
rotlet, stresslet, quadrupole and octupole of the multipole moment method (vide infra). This suggests an inherent deficiency in the multipole collocation technique for nonspherical particles, since even an isolated ellipsoid creates a disturbance which can be correctly reproduced only by an infinite series of Stokes singularities at its geometric centre (Brenner 1964b).

By allowing the singularities to be distributed within the particle however (rather than restricting their location to the middle), Chwang \& Wu (1975) constructed exact solutions for isolated spheroids in linear ambient flows using only low-order singularities such as Stokeslets and doublets, rotlets, and stresslets and octupoles. Their singularity representation, reminiscent of the slender-body theory pioneered by Batchelor (1970), is much more convenient for numerical purposes than the grantedly more compact symbolic operator formalism of Brenner \& Haber (1983) (see also Brenner 1966), which, in effect, places an infinite series of disturbances at the centre of the particle, just as in the multipole collocation technique. Kim (1985b) furnished a very elegant proof of the functional equivalence between Faxén laws and the singularity representation of disturbance velocities for rigid particles in Stokes flow. (Kim \& Lu 1987 extended their theorem to include fluid-fluid boundaries.) He then used Chwang \& Wu's non-local formulation for spheroids $(1974,1975)$ in conjunction with the method of reflections to examine the sedimentation of two identical spheroids in an unbounded fluid (Kim 1985a). Since the formulation is exact in the absence of interactions (unlike the multipole collocation approach for non-spherical bodies), better accuracy is expected for the same number of unknown multipoles, even though many iterations may be necessary. Kim carried the calculations out to the second reflection, but lost convergence at small separations.

The multipole collocation and the multipole moment methods are similar in many ways and have been blended in a few hybrid approaches. Indeed, for spheres, as pointed out, there exists a one-to-one correspondence between the disturbances of the collocation technique and the stress moment propagators of the moment expansion. Yet this second scheme is substantially more efficient and typically introduces fewer unknowns. It also very readily accommodates periodic boundary conditions to simulate suspensions of infinite extent. The multipole-moment technique expands the contribution of the surface stresses on each particle in the boundary integral equation (1.2) into moments about the particle centre. The zeroth moment singles out the effect of the total force. The first moment has antisymmetric and symmetric components. The former corresponds to the net torque experienced by the particle; the latter is identified as the stresslet. Higher moments yield the quadrupole, octupole, hexadecupole, etc. This series is truncated at the level appropriate for the accuracy desired. The motion of each submerged object is then determined from Faxén formulae, with the velocity field given by the moments expansion of (1.2). This method lies at the core of Stokesian dynamics (Brady \& Bossis 1988; Brady et al. 1988) and of the technique presented herein, and will therefore be explained in greater detail in $\$ 2$. The only application to non-spherical particles of a variant of the multipole moment method of which we are aware, is the work by Yoon \& Kim (1990), in which up to four moments are retained to describe the dynamics of pairs of spheroids. Instead of relying of Faxén relations, a least-squares collocation technique is used to calculate the strength of the unknown multipoles from the no-slip boundary conditions.

Stokesian dynamics, by introducing at most 11 unknowns per particle, is a very costefficient numerical simulation technique for suspensions of spheres. It combines the multipole moment method with lubrication formulae to remedy the inadequacy of the moments expansion when particles are nearly touching. It is therefore accurate over 
the full concentration range, from very dilute to maximum packing. In addition, the method consistently preserves any symmetry inherent to the system by making explicit use of Lorentz' reciprocal theorem (Brenner 1963; Brenner 1964b; Hinch 1972; Claeys \& Brady 1989) in order to improve both its numerical efficiency and its robustness. Since our approach for prolate spheroids essentially adopts the same strategy, we discuss Stokesian dynamics in the next section. Section 3 then details how far-field interactions are calculated (i.e. the multipole moment method for our system), and near-field aspects are tackled in $\S 4$. The accuracy and efficiency of the method is illustrated by means of several examples in $\S 5$. We summarize our main findings in the concluding section.

\section{Stokesian dynamics for a finite number of particles}

Two basic problem formulations exist in suspension mechanics. In the mobility version, the velocities (both translational and rotational) of the particles are sought, given the ambient flow field and the forces and torques acting on each body. The converse, finding the forces and torques required to impose a desired particulate motion in an external flow, is referred to as the resistance problem. The governing equations (1.1) are solved subject to no-slip boundary conditions

$$
u(x)=U_{p}+\left(x-x_{p}\right) \wedge \Omega_{p}, \quad x \in A_{p}
$$

on the surface $A_{p}$ of each particle (labelled by integers $p$ from 1 to $N$ ). $\Omega_{p}$ denotes the body's angular velocity, and $U_{p}$ is the translational velocity of its locator point $\boldsymbol{x}_{p}$ (usually its centre of hydrodynamic stress (Brenner 1963)). Far away from the cluster, the imposed linear flow $\boldsymbol{u}_{\infty}$ with uniform velocity $\boldsymbol{U}_{\infty}$, vorticity $\boldsymbol{\Omega}_{\infty}$, and rate of strain $E_{\infty}$ must be recovered:

$$
\boldsymbol{u}(\boldsymbol{x}) \rightarrow \boldsymbol{u}_{\infty}(x)=U_{\infty}+x \wedge \Omega_{\infty}+E_{\infty} \cdot x, \quad|x| \rightarrow \infty .
$$

Owing to the linearity of the boundary conditions and of the Stokes equations, the forces and torques exerted by the particles on the fluid are related through tensors to their velocities and angular velocities relative to the undisturbed flow and to the imposed rate of strain. For instance, the force $F_{p}$ on particle $p$ may be found as

$$
F_{p}=\sum_{q-1}^{N} R_{F U}^{p q} \cdot\left(U_{q}-u_{\infty}\left(x_{q}\right)\right)+\sum_{q=1}^{N} R_{F \Omega}^{p q} \cdot\left(\Omega_{q}-\Omega_{\infty}\right)-\sum_{q-1}^{N} R_{F E}^{p q}: E_{\infty}
$$

The resistance tensor $\boldsymbol{R}_{F U}^{p q}$ gives the force experienced by particle $p$ due to a translation of $q$, holding all objects other than $q$ fixed. (Its evaluation therefore requires the solution of an $N$-body problem.) The meaning of all other quantities in (2.3) should be clear. Relations such as these may be written down for the torques, the stresslets, the octupoles, and all higher moments of the force density on the surface of the particles. Grouping the forces and torques of all bodies into a vector $\mathscr{F}$ of length $6 \mathrm{~N}$, their translational and angular velocities into $\mathscr{U}$, and the undisturbed fluid velocity and vorticity at their locator points into $\mathscr{U}_{\infty}$, we may write the resistance problem as:

$$
\mathscr{F}=\mathscr{R}_{\mathscr{F} \Psi} \cdot\left(\mathscr{U}-\mathscr{U}_{\infty}\right)-\mathscr{R}_{\mathscr{F} E}: \boldsymbol{E}_{\infty} \text {. }
$$

Similarly, if the stresslets of all $N$ particles are stringed in $\mathscr{S}$,

or, in matrix notation,

$$
\begin{gathered}
\mathscr{S}=\mathscr{R}_{S \mathscr{U}} \cdot\left(\mathscr{U}-\mathscr{U}_{\infty}\right)-\mathscr{R}_{S E}: E_{\infty}, \\
\left(\begin{array}{c}
\mathscr{F} \\
\mathscr{S}
\end{array}\right)=\mathscr{R} \otimes\left(\begin{array}{c}
\mathscr{U}-\mathscr{U}_{\infty} \\
-E_{\infty}
\end{array}\right) .
\end{gathered}
$$

(The operator $\otimes$ signifies the appropriate contraction.) 
Dynamic simulations require one to solve (2.6) for the velocities $\mathscr{U}$ and integrate the trajectories over a small time-step to find the evolution of the particle configuration. The resistance tensor is then constructed anew with the updated particle locations. The dynamics associated with Brownian motion are well understood (Ermak \& McCammon 1978) and can be incorporated rigorously. (This is explained for spherical particles in Brady \& Bossis 1988. The thermal motions of prolate spheroids can be treated in a similar fashion.)

Pairwise additive schemes construct the resistance tensors, or their inverse, by considering all pairs of particles successively and calculating their interactions as if all other bodies were absent. In effect, in the resistance formulation, the $\boldsymbol{R}^{p q}$ (see (2.3)) are evaluated as two-body characteristics rather than properties depending on the configuration of all $N$ particles. Since the first many-body effects enter the mobility formulae at $O\left(r^{-4}\right)$, with $r$ the centre-to-centre distance between particles (Mazur \& van Saarloos 1982), while third-body reflections contribute terms of $O\left(r^{-2}\right)$ to the resistance problem, the pairwise mobility formulation is more accurate than the resistance version (additivity of forces). In essence, any pair of particles senses the presence of other objects less when these are freely suspended than when they are fixed in space. Moreover, the series expansion in $r^{-1}$ for the mobility functions converges more rapidly than the series for the resistance or friction tensors (Felderhof 1977). Thus pairwise additivity of velocities is preferable to additivity of forces. Lubrication forces, however, are only preserved in the resistance approach. (The tensor $\boldsymbol{R}_{F U}^{p q}$ for two touching particles $p$ and $q$ is singular and therefore dominates the behaviour. In contrast, its inverse vanishes, and its effect will be swamped by that of all other particles, leading to particle overlap in any scheme using pairwise additivity of velocities (Bossis \& Brady 1984).) These observations lie at the heart of the method called Stokesian dynamics (Durlofsky, Brady \& Bossis 1987). The $N$-body mobility tensor is first approximated using the more accurate pairwise additivity of velocities. It is then inverted to yield a 'far-field' estimate of the resistance tensor, to which lubrication stresses are added using the two-body resistance functions. Some multipole reflections between nearly touching objects may be included in both the mobility inverse and the lubrication tensor, and must be subtracted in a corrective step. In summary,

$$
\mathscr{R} \approx \boldsymbol{R}=\boldsymbol{M}^{-1}+\mathbf{R}_{\mathrm{lub}}-\mathbf{R}_{\text {corr }} .
$$

As shown by Durlofsky et al. (1987) and by Mazur (1982), the inversion of the $N$-body mobility tensor $\boldsymbol{M}$ sums all the reflected interactions between all moments retained during its construction. The inverse $\boldsymbol{M}^{-1}$ therefore contains many-body effects, and $\boldsymbol{R}$ is a better estimate of $\mathscr{R}$ than what would have been obtained by pairwise additivity of forces. Furthermore, by adding the very strong and localized interactions $\mathbf{R}_{\text {lub }}$ between nearly touching particles, the algorithm of Stokesian dynamics also preserves lubrication interactions.

The mobility tensor $M$ is constructed by generalizing the procedure originally applied to dispersions of spheres by Durlofsky et al. (1987) (Claeys 1991). The velocity disturbance due to each particle is first decomposed into the contributions of the irreducible moments of the stress density on its surface: at the zeroth order, the effect of the force exerted by the particle on the fluid is quantified; at the next level, the perturbation due to the first moment is evaluated. This comprises an antisymmetric part related to the torque, and a symmetric component which is the stresslet:

$$
S_{q}=-\int_{A_{q}} \frac{1}{2}\left[n \cdot \sigma\left\{y-x_{q}\right\}+\left(y-x_{q}\right) \sigma \cdot n\right]-\frac{1}{3} \delta\left[n \cdot \sigma \cdot\left(y-x_{q}\right)\right] \mathrm{d} A_{y},
$$


with $n$ the unit outward normal to the surface $A_{q}$. This is continued to any order for the accuracy desired. In most applications of Stokesian dynamics (Brady \& Bossis 1988; Phillips, Brady \& Bossis 1988 a,b; Bossis \& Brady 1989), the multipole expansion of the velocity disturbance has been truncated after the first two terms, retaining the effects of the force, the torque and the stresslet. A mean-field estimate of the quadrupolar contribution has sometimes been included also. Those simulations produce results in excellent agreement with experimental data.

In the algorithm of Stokesian dynamics, the impact of the velocity disturbance on the motion of all other particles is determined by applying generalized Faxén laws. These relations express the motion of a freely suspended object as a function of the unperturbed fluid velocity field in which it is immersed. Each element of the mobility tensor thus isolates the effect of one irreducible stress moment of a particle ' $p$ ' (the force it exerts on the liquid for instance) on one component of the motion of a particle ' $q$ ' (its angular velocity for example). This procedure, sketched in general terms above, is applied to prolate spheroids in the next section.

\section{Far-field estimate of the resistance tensor for prolate spheroids}

The far-field estimate of the resistance tensor is the many-body approximation to $\mathscr{R}$ obtained by inverting the grand mobility tensor $\boldsymbol{M}$. The previous section briefly outlines how to construct $\boldsymbol{M}$ by combining the Faxén relations for the particles with the irreducible multipole expansion of the fluid velocity disturbance due to their presence in the flow. Although our work primarily concerns prolate spheroids, some of the results reported in this section are presented for arbitrary ellipsoids. It is not difficult to take the limit for ovary ellipsoids of revolution if so desired.

For a generic ellipsoid $p$ centred at $x_{p}$ and with surface $A_{p}$ such that

$$
x \in A_{p} \Leftrightarrow A_{p}^{-1}:\left(x-x_{p}\right)\left(x-x_{p}\right)=1,
$$

with $\boldsymbol{A}_{p}$ a positive-definite symmetric tensor of rank two, we show in Appendix $A$ that the disturbance velocity field can be decomposed into contributions from irreducible multipoles $I_{p}^{(m)}$ as follows:

$$
u(x)-u_{0}(x)=\frac{1}{8 \pi \mu} \sum_{m=0}^{\infty} I_{p}^{(m)} \odot^{m+1}(2 m+1) ! !\left[\left(\frac{1}{\mathrm{D}_{p}} \frac{\mathrm{d}}{\mathrm{dD}_{p}}\right)^{m} \frac{\sinh \mathrm{D}_{p}}{\mathrm{D}_{p}}\right] \overbrace{x_{p}}^{\times m} J\left(x-x_{p}\right) .
$$

The velocity field $\boldsymbol{u}_{0}$ is the undisturbed flow as it would be in the absence of the particle. The scalar operator $D_{p}=\left(D_{p}^{2}\right)^{\frac{1}{2}}$, with

$$
\mathrm{D}_{p}^{2}=\boldsymbol{A}_{p}: \nabla \nabla=\sum_{i=1}^{3} a_{i}^{2} e_{i} e_{i}: \nabla \nabla=\sum_{i=1}^{3} a_{i}^{2} \frac{\partial^{2}}{\partial x_{i}^{2}}
$$

where we have defined an orthogonal coordinate system $\left(x_{1}, x_{2}, x_{3}\right)$ using the eigenvectors $e_{i}$ of $\boldsymbol{A}_{p}$. These lie along the principal axes of the ellipsoid, and we shall assume that they have been labelled such that $a_{1} \geqslant a_{2} \geqslant a_{3}$. As usual, ( $\left.\sinh \mathrm{D}_{p}\right) / \mathrm{D}_{p}$ must be interpreted as its infinite series representation (with $\mathrm{D}_{p}^{4}=\mathrm{D}_{p}^{2} \mathrm{D}_{p}^{2}$, etc.) and $(2 m+1) ! !=(2 m+1) ! /\left(2^{m} m !\right)$. The irreducible stress moments $I_{p}^{(m)}$ are defined for ellipsoids so that contractions of any two of their last $m$ indices with the dyadic $\boldsymbol{A}_{p}^{-1}$ yield zero (cf. Appendix A). We have also introduced the notations $\overbrace{t}^{x_{m}}$ for the direct product of $m$ tensors $t$ (or tensorial operators $t$ ), and $\odot^{m}$ for the $m$-fold contraction operator (for which we adopt the 'nesting' convention; see, for example, Chapman \& Cowling 1970). 
Faxén laws for the stress moments were derived by Kim \& Arunachalam (1987). At the two lowest orders, their results can be cast in the form:

$$
\begin{gathered}
F_{p}=I_{p}^{(0)}=K_{F U}^{p} \cdot U_{p}-\left.K_{F U}^{p} \cdot\left[\left(\frac{\sinh \mathrm{D}_{p}}{\mathrm{D}_{p}}\right) u_{0}(x)\right]\right|_{x=x_{p}}, \\
I_{p}^{(1)}=Z^{p}: \varepsilon \cdot \Omega_{p}-Z^{p}:\left.\left[3\left(\frac{1}{\mathrm{D}_{p}} \frac{\mathrm{d}}{\mathrm{dD}_{p}} \frac{\sinh \mathrm{D}_{p}}{\mathrm{D}_{p}}\right) \nabla u_{0}(x)\right]\right|_{x=x_{p}},
\end{gathered}
$$

where $\varepsilon$ represents the alternating triadic. The polyadics $K_{F U}^{p}$ and $Z^{p}$ can be expressed in terms of elliptic integrals depending only on the body's dimensions and the orientation of its principal axes (Brenner 1966; Rallison 1978; Brenner \& Haber 1983). It is now easy, at least conceptually, to build the grand mobility tensor for a system of arbitrary ellipsoids by combining the irreducible expansion for the velocity disturbance (3.2) with the resistance functions (3.4). The tensor $M_{U F}^{p q}$ for example, which links the translational velocity of particle $p$ to the force exerted on the fluid by ellipsoids $q$, can be written as (for $p \neq q$ ):

$$
M_{U F}^{p q}=\frac{1}{8 \pi \mu}\left(\frac{\sinh \mathrm{D}_{p}}{\mathrm{D}_{p}}\right)\left(\frac{\sinh \mathrm{D}_{q}}{\mathrm{D}_{q}}\right) \mathcal{J}\left(x_{p}-x_{q}\right)
$$

An alternative, non-local description of the particle's interactions with the flow field exists. Kim (1985b) showed that the Faxén relations for a prolate spheroid can be expressed as integrals of the velocity field $\boldsymbol{u}_{0}$ and of its gradients over a line segment along the spheroidal symmetry axis connecting the foci at $x_{p} \pm c_{p} d_{p}$. His results (for the force, torque and stresslet only) read: $\dagger$

$$
\begin{aligned}
F= & 16 \pi \mu c\left\{\alpha_{1} d d+\alpha_{2}(\delta-d d)\right\} \cdot U \\
& -8 \pi \mu\left\{\alpha_{1} d d+\alpha_{2}(\delta-d d)\right\} \cdot \int_{-c}^{c}\left\{1+\left(c^{2}-\xi^{2}\right) \frac{1-e^{2}}{4 e^{2}} \nabla^{2}\right\} u_{0}(\xi) \mathrm{d} \xi \\
T= & \frac{32}{3} \pi \mu c^{3}\left\{\gamma d d+\gamma^{\prime}(\delta-d d)\right\} \cdot \Omega \\
& -4 \pi \mu\left\{\gamma d d+\gamma^{\prime}(\delta-d d)\right\} \cdot \int_{-c}^{c}\left(c^{2}-\xi^{2}\right) \nabla \wedge u_{0}(\xi) \mathrm{d} \xi \\
& -8 \pi \mu \alpha_{1} d \wedge \int_{-c}^{c}\left(c^{2}-\xi^{2}\right)\left\{1+\left(c^{2}-\xi^{2}\right) \frac{1-e^{2}}{8 e^{2}} \nabla^{2}\right\} d \cdot e_{0}(\xi) \mathrm{d} \xi \\
S_{i j}= & 8 \pi \mu\left\{\frac{\alpha_{5}}{2}\left(d_{i} d_{j}-\frac{1}{3} \delta_{i j}\right)\left(d_{k} d_{l}-\frac{1}{3} \delta_{k l}\right)\right. \\
& +\frac{1}{4} \alpha^{*}\left(d_{i} \delta_{j k} d_{l}+d_{i} \delta_{j l} d_{k}+\delta_{i l} d_{j} d_{k}+\delta_{i k} d_{j} d_{l}-4 d_{i} d_{j} d_{k} d_{l}\right) \\
& +\frac{1}{2} \alpha_{4}\left(\delta_{i k} \delta_{j l}+\delta_{i l} \delta_{j k}-\delta_{i j} \delta_{k l}+d_{i} d_{j} \delta_{k l}+\delta_{i j} d_{k} d_{l}\right. \\
& \left.-d_{i} \delta_{j l} d_{k}-\delta_{i k} d_{j} d_{l}-d_{i} \delta_{j k} d_{l}-\delta_{i l} d_{j} d_{k}+d_{i} d_{j} d_{k} d_{l}\right\} \\
& \times \int_{-c}^{c}\left(c^{2}-\xi^{2}\right)\left\{1+\left(c^{2}-\xi^{2}\right) \frac{1-e^{2}}{8 e^{2}} \nabla^{2}\right\}\left[\theta_{0}(\xi)\right]_{k l} \mathrm{~d} \xi \\
& -2 \pi \mu \alpha_{1}\left(d_{i} \epsilon_{j k l} d_{l}+d_{j} \epsilon_{i k l} d_{l}\right) \int_{-c}^{c}\left(c^{2}-\xi^{2}\right)\left\{\nabla \wedge u_{0}(\xi)-2 \Omega\right\}_{k} \mathrm{~d} \xi
\end{aligned}
$$

$\dagger$ Kim \& Arunachalam (1987) later generalized these formulae to any multipole moment for arbitrary ellipsoids. 
(All variables, except $\mu$, relate to particle $p$. We suppressed the subscript $p$ on $F, T, S$, $\boldsymbol{U}, \boldsymbol{\Omega}, \boldsymbol{d}, \boldsymbol{\alpha}, \boldsymbol{\gamma}, c$ and $e$ for clarity. The implicit summation convention for repeated indices is adopted in (3.6c).) The unit vector $d_{p}$ (for director) lies along the spheroidal axis; $U_{p}$ and $\boldsymbol{\Omega}_{p}$ are the translational and angular velocities of the particle at its locator point $x_{p}$. The notation $\boldsymbol{e}_{0}$ is shorthand for $\frac{1}{2}\left(\nabla u_{0}+{ }^{\mathrm{T}} \nabla u_{0}\right)$, and $\xi$ stands for $x_{p}+\xi d_{p}$. All the remaining parameters depend only on the geometry of the body: $c$ is the distance between the geometric centre and the foci of the generating ellipse; $e=c / a$ is called the eccentricity, with $a$ the length of the major semi-axis; $\alpha$ and $\gamma$ are functions of $e$, defined in Kim (1985b) (see also Chwang \& Wu 1974, 1975, and Appendix B). In essence, $\alpha_{5}$ dominates during axisymmetric extension about the spheroidal axis, $\alpha^{*}$ characterizes the stress response to simple shear flows for which the velocity gradient lies along $d_{p}$, and $\alpha_{4}$ gives the particle's stress contribution when the plane of shear is perpendicular to the director.

The two formulations (3.4) and (3.6) are of course compatible, since one can show for any tensor $\boldsymbol{X}(\boldsymbol{x})$ that (Kim \& Arunachalam 1987)

$$
\begin{aligned}
& \left.\left(\frac{1}{\mathrm{D}} \frac{\partial}{\partial \mathrm{D}}\right)^{m} \frac{\sinh \mathrm{D}}{\mathrm{D}} X(x)\right|_{x=x_{p}} \\
& \quad=\frac{1}{2 \pi c_{E} c_{E}^{\prime}} \frac{1}{(2 m-1) ! !} \iint_{E} q^{2 m-1}\left(x^{\prime}\right)\left\{1+\frac{a_{3}^{2} q^{2}}{4 m+2} \nabla^{2}\right\} X\left(x^{\prime}\right) \mathrm{d} A_{x^{\prime}} .
\end{aligned}
$$

The domain of integration $E$ is the elliptical disk confocal to the particle surface $A_{p}$ :

$$
x \in E \Leftrightarrow q^{2}(x)=1-\frac{\left[\left(x-x_{p}\right) \cdot e_{1}\right]^{2}}{c_{E}^{2}}-\frac{\left[\left(x-x_{p}\right) \cdot e_{2}\right]^{2}}{c_{E}^{\prime 2}}>0,
$$

with $c_{E}^{2}=a_{1}^{2}-a_{3}^{2}$ and $c_{E}^{\prime 2}=a_{2}^{2}-a_{3}^{2}$. For a prolate spheroid, with $a_{2}=a_{3}$ and $e_{1}=d$, the fundamental ellipse degenerates into a line with length $2 c_{E}=2 c$, and (3.7a) takes the form:

$$
\begin{aligned}
& \left.\left(\frac{1}{\mathrm{D}} \frac{\partial}{\partial \mathrm{D}}\right)^{m} \frac{\sinh \mathrm{D}}{\mathrm{d}} X(x)\right|_{x-x_{p}} \\
& \quad=\frac{1}{2 c} \int_{-c}^{c} \frac{1}{2^{m} m !}\left(1-\frac{\xi^{2}}{c^{2}}\right)^{m}\left\{1+\frac{a_{3}^{2}}{4 m+4}\left(1-\frac{\xi^{2}}{c^{2}}\right) \nabla^{2}\right\} X\left(x_{p}+\xi d_{p}\right) \mathrm{d} \xi
\end{aligned}
$$

Note that $a_{3}^{2} / c^{2}=\left(1-e^{2}\right) / e^{2}$ and compare (3.7c) with (3.6).

The identity (3.7a) can be used in conjunction with the irreducible expansion (3.2) to write the disturbance velocity as

$$
\begin{aligned}
& u(x)-u_{0}(x) \\
& =\frac{1}{8 \pi \mu} \sum_{m=0}^{\infty} I_{p}^{(m)} \odot^{m+1} \frac{2 m+1}{2 \pi c_{E} c_{E}^{\prime}} \iint_{E} q^{2 m-1}\left(x^{\prime}\right)\left\{1+\frac{a_{3}^{2} q^{2}}{4 m+2} \nabla^{2}\right\} \widetilde{\nabla}_{x^{\prime}}^{\times m} J\left(x-x^{\prime}\right) \mathrm{d} A_{x^{\prime}} .
\end{aligned}
$$

This is consistent with the singularity representation of Chwang \& Wu $(1974,1975)$, who constructed exact solutions of the Stokes equations for isolated prolate spheroids suspended in linear flows using uniform, parabolic and biquadratic distributions of singularities along the particle's major axis.

We now have all the elements necessary to build the grand mobility tensor, but need to invert the Faxén laws (3.6). It is trivial to obtain

$$
U-u_{\infty}\left(x_{p}\right)=\frac{1}{16 \pi \mu c}\left\{\alpha_{1}^{-1} d d+\alpha_{2}^{-1}(\delta-d d)\right\} \cdot F+\frac{1}{2 c} \int_{-c}^{c}\left\{1+\left(c^{2}-\xi^{2}\right) \frac{1-e^{2}}{4 e^{2}} \nabla^{2}\right\} u^{\prime}(\xi) d \xi
$$


where we have extracted the contribution from the impressed flow $\boldsymbol{u}_{x}$ (see (2.2)), and introduced $u^{\prime}=u_{0}-u_{\infty}$. (In Stokesian dynamics, $u^{\prime}$ typically represents the disturbance velocity due to all particles other than $p$.) The relations $(3.6 b, c)$ for $\boldsymbol{\Omega}-\boldsymbol{\Omega}_{\infty}$ and $-\boldsymbol{E}_{\infty}$, however, are coupled. This reflects the fact that a spheroid tumbles in an extensional flow (unless its axis is aligned with a principal direction of the rate of strain). The expressions $(3.6 b, c)$ must therefore be inverted together. After a bit of algebra (Claeys 1988), one arrives at the following mobility functions for prolate spheroids (see also Kim 1986):

$$
\begin{aligned}
\boldsymbol{\Omega}-\boldsymbol{\Omega}_{\infty}= & \frac{3}{32 \pi \mu c^{3}}\left\{\gamma^{-1} d d+\left[\gamma^{\prime-1}-\frac{e^{2} /\left(2-e^{2}\right)}{\left(\left(2-e^{2}\right) / e^{2}\right) \alpha^{*}+\alpha_{1}}\right](\delta-d d)\right\} \cdot \boldsymbol{T} \\
& +\frac{3}{32 \pi \mu c^{3}} \frac{1}{\left(\left(2-e^{2}\right) / e^{2}\right) \alpha^{*}+\alpha_{1}}(d \wedge \boldsymbol{S} \cdot \boldsymbol{d}-\boldsymbol{d} \cdot \boldsymbol{S} \wedge d) \\
& +\frac{3}{8 c^{3}} \int_{-c}^{c}\left(c^{2}-\xi^{2}\right) \nabla \wedge \boldsymbol{u}^{\prime}(\xi) \mathrm{d} \xi
\end{aligned}
$$

and

$$
\begin{aligned}
-\left(\boldsymbol{E}_{x}\right)_{i j}= & \frac{-3}{32 \pi \mu c^{3}}\left\{\frac{9}{2 \alpha_{5}}\left(d_{i} d_{j}-\frac{1}{3} \delta_{i j}\right)\left(d_{k} d_{l}-\frac{1}{3} \delta_{k l}\right)\right. \\
& +\frac{1}{\alpha^{*}+\left(e^{2} /\left(2-e^{2}\right)\right) \alpha_{1}}\left(d_{i} \delta_{j k} d_{l}+d_{i} \delta_{j l} d_{k}+\delta_{i l} d_{j} d_{k}+\delta_{i k} d_{j} d_{l}-4 d_{i} d_{j} d_{k} d_{l}\right) \\
& +\frac{1}{2 \alpha_{4}}\left(\delta_{i k} \delta_{j l}+\delta_{i l} \delta_{j k}-\delta_{i j} \delta_{k l}+d_{i} d_{j} \delta_{k l}+\delta_{i j} d_{k} d_{l}\right. \\
& \left.\left.-d_{i} \delta_{j l} d_{k}-\delta_{i k} d_{j} d_{l}-d_{i} \delta_{j k} d_{l}-\delta_{i l} d_{j} d_{k}+d_{i} d_{j} d_{k} d_{l}\right)\right\} S_{k l} \\
& +\frac{3}{4 c^{3}} \int_{-c}^{c}\left(c^{2}-\xi^{2}\right)\left\{1+\left(c^{2}-\xi^{2}\right) \frac{1-e^{2}}{8 e^{2}} \nabla^{2}\right\} e_{i j}^{\prime}(\xi) \mathrm{d} \xi \\
& -\frac{3}{32 \pi \mu c^{3}} \frac{1}{\left.\left(2-e^{2}\right) / e^{2}\right) \alpha^{*}+\alpha_{1}}(d d \wedge T-T \wedge d d)_{i j}
\end{aligned}
$$

As before, $e^{\prime}=\frac{1}{2}\left(\nabla u^{\prime}+{ }^{\mathrm{T}} \nabla u^{\prime}\right)$. It may be useful to point out that

$$
e^{2} /\left(2-e^{2}\right)=\left(r_{p}^{2}-1\right) /\left(r_{p}^{2}+1\right),
$$

with $r_{p}$ the aspect ratio of the particle $\left(r_{p}>1\right.$ for prolate bodies).

From the linearity of the creeping flow equations, $\boldsymbol{u}^{\prime}$ may be constructed by superposing the contributions (3.8) of each particle separately. Truncating the expansion after the first moment (experience with Stokesian dynamics for spheres indicates that retaining the effects of the force, torque and stresslet is sufficient for most purposes), we rewrite (3.8) explicitly for prolate spheroids:

$$
\begin{aligned}
u^{\prime}(x)= & \frac{1}{16 \pi \mu c} F_{q} \cdot \int_{-c}^{c}\left\{1+\left(c^{2}-\xi^{2}\right) \frac{1-e^{2}}{4 e^{2}} \nabla^{2}\right\} J(x-\xi) \mathrm{d} \xi \\
& -\frac{3}{64 \pi \mu c^{3}} T_{q} \cdot \int_{-c}^{c}\left(c^{2}-\xi^{2}\right) \nabla_{x} \wedge J(x-\xi) \mathrm{d} \xi \\
& -\frac{3}{32 \pi \mu c^{3}} S_{q}: \int_{-c}^{c}\left(c^{2}-\xi^{2}\right)\left\{1+\left(c^{2}-\xi^{2}\right) \frac{1-e^{2}}{8 e^{2}} \nabla^{2}\right\} \frac{1}{2}\left(\nabla_{x}+{ }^{\mathrm{T}} \nabla_{x}\right) J(x-\xi) \mathrm{d} \xi .
\end{aligned}
$$


The grand mobility tensor for a system of $N$ prolate spheroids can be constructed at the level of forces, torques and stresslets by summing the disturbances (3.10) over all particles $q \neq p$, inserting the result into the mobility functions (3.9), and repeating this for all spheroids $p$. (The inclusion of higher moments is straightforward provided their Faxén laws are first determined.) The contribution of a force $F_{2}$ exerted by particle ' 2 ' to the translational velocity $U_{1}$ of spheroid ' 1 ', for example, is given by

$$
\begin{aligned}
U_{1}=\frac{1}{32 \pi \mu c_{1} c_{2}} \int_{-c_{1}}^{c_{1}}\left[\left\{1+\left(c_{1}^{2}-\xi_{1}^{2}\right) \frac{1-e_{1}^{2}}{4 e_{1}^{2}} \nabla^{2}\right\}\right. \\
\left.\qquad \int_{-c_{2}}^{c_{2}}\left\{1+\left(c_{2}^{2}-\xi_{2}^{2}\right) \frac{1-e_{2}^{2}}{4 e_{2}^{2}} \nabla^{2}\right\} J\left(\xi_{1}-\xi_{2}\right)\right] \mathrm{d} \xi_{2} \mathrm{~d} \xi_{1} \cdot F_{2} .
\end{aligned}
$$

In contrast to (3.5), this non-local description of the interactions between spheroids only involves the Oseen tensor and its second derivative (recall that $\nabla^{4} J=0$ ). Equation (3.11) shows that the rods behave hydrodynamically as if they were line distributions of point forces and potential doublets - no higher multipoles are needed for the $M_{U F}^{p q}$-coupling. This is a definite advantage over the symbolic representation (3.5) since multipoles of higher order generate more complex flow disturbances and have stronger singularities at their origin. Indeed, we have found the formulation (3.5) to be totally unsatisfactory for numerical purposes in cases where the centre-to-centre separation of the particles is comparable to the sum of their major semi-axes (i.e. when the spheres circumscribing the spheroids penetrate). In (3.5), mobility elements are calculated as infinite alternating series with very poor convergence properties at small distances. The non-local description (3.11) is far more robust, although the kernel can be sharply peaked or rapidly varying for nearly touching particles. Adaptive integration techniques (Piessens et al. 1983) handle these numerical difficulties very nicely. The mobility tensor $\boldsymbol{M}$ obtained in this way can be inverted using a Cholesky decomposition algorithm (see for example Perry \& Green $1984, \S 2$ ) since $M$ is positive definite, a consequence of viscous dissipation, and also symmetric from Lorentz' reciprocal theorem (Happel \& Brenner 1973).

The computation of each element of the mobility tensor (except the self-terms $\boldsymbol{M}^{p p}$ ) requires a double integration over the particle axes. Using cylindrical coordinates centred at the origin of spheroid 2 with the azimuthal axis along $d_{2}$, the first integration in (3.11) can be carried out analytically. The result is most conveniently expressed in terms of the coefficients $B_{m, n}(x)$, which Chwang \& Wu (1975) introduced as:

$$
B_{m, n}(x)=\int_{-c_{2}}^{c_{2}} \frac{\xi^{n}}{|x-\xi|^{m}} \mathrm{~d} \xi .
$$

For instance, defining a right-handed orthonormal frame of reference $\left(d_{2}, \boldsymbol{e}_{r}, \boldsymbol{e}_{\theta}\right)$ such that $x-x_{2}=x_{d} d_{2}+r e_{r}$, we find

$$
\begin{aligned}
\int_{-c_{2}}^{c_{2}} J\left(x-\xi_{2}\right) \mathrm{d} \xi_{2}=\left(2 B_{1,0}-r^{2} B_{3,0}\right) d_{2} d_{2} & +\left(x_{d} B_{3,0}-B_{3,1}\right) r\left(d_{2} e_{r}+e_{r} d_{2}\right) \\
& +\left(B_{1,0}+r^{2} B_{3,0}\right) e_{r} e_{r}+B_{1,0} e_{\theta} e_{\theta} .
\end{aligned}
$$

Note that $x=\xi_{1}$ in (3.11). Therefore, $e_{r}$ and $e_{\theta}$ are functions of $\xi_{1}$, whose variation must be taken into account during the subsequent integration along the axis of spheroid 1. 
Closed-form expressions for $B_{m, n}(x)$ in terms of elementary functions are known for arbitrary integer values of $m$ and $n$, and recurrence formulae for $n>1$ (Chwang \& Wu 1975; Claeys 1988) make their use especially suited for computer programming. Taking advantage of identities relating derivatives of $B_{m, n}(x)$ (Claeys 1988), and after some tedious but straightforward algebra, all the mobility coefficients for particles 1 and 2 can be written as integrals along the symmetry axis of either ellipsoid in the form $\mu^{-1} \int_{-c_{1}}^{c_{1}} f_{M}\left(\left\{B_{m, n}\left(\xi_{1}\right)\right\}\right) \mathrm{d} \xi_{1}$, where $f_{M}\left(\left\{B_{m, n}\right\}\right)$ is a linear function of the set of $B_{m, n}(x)$ involving $c_{1}, c_{2}, e_{1}$ and $e_{2}$ as parameters. This integral is then evaluated numerically (Claeys 1988, 1991).

\section{Addition of lubrication effects}

As explained in $\$ 2$, lubrication stresses arising from the relative motion of nearly touching particles in the fluid are added in a pairwise manner to the mobility invert. Unlike the far-field hydrodynamic interactions, lubrication is essentially a two-body problem dominated by the flow in the small and narrow gap region separating the close surfaces. Hence pairwise additive schemes should be successful. The construction of the resistance tensor for two objects near contact involves two steps. At first, the points of closest approach must be determined. This then specifies the local geometry in the thin gap, from which the friction functions can be calculated. Claeys \& Brady (1989) derived formulae for all stress moments which diverge as the surface separation $\epsilon$ vanishes; i.e. the resistance tensor is known to $O\left(\epsilon^{0}\right)$ for arbitrary objects (with mathematically smooth boundaries which would touch at a single point when brought into contact along their common normal without altering their orientation - all convex particles satisfy these conditions). The $O(1)=O\left(\epsilon^{0}\right)$ corrections to these expressions require the solution of the 'outer' flow, which is quite complex and depends on the exact relative position of the particles (characterized by four independent parameters for axisymmetric bodies). Although they can be found using collocation techniques or a finite elements method for example, even the task of tabulating them is forbidding.

Since the points of minimum separation $z_{p}$ and $z_{q}$ on particles $p$ and $q$ must be determined over and over during dynamic simulations, it is imperative to develop an efficient and accurate algorithm devoted to this goal. Precision is important since even rather good approximations to the points of closest approach can give poor estimates of the gap width $\epsilon$, with which the resistance functions scale (figure $1 a$ ). In addition, in the acicular limit, the principal radii of curvature, which also largely determine the magnitude of the stress response, change very rapidly near the tip of the rod (figure $1 b$ ). Thus the algorithm must converge very quickly to the exact location of $z_{p}$ and $z_{q}$. The method we have devised is based on the observation that the normal to the surface $A_{p}$ at $z_{p}$ coincides with the normal to $A_{q}$ at $z_{q}$ (but is antiparallel to it). We also exploit the following property of ellipses: the sum of the distances from any point $x$ of the ellipse to the foci is constant. The surface $A_{p}$ of a prolate spheroid centred at the origin is thus correctly represented by

$$
x \in A_{p} \Leftrightarrow f_{p}(x)=\left|x-c_{p}\right|+\left|x+c_{p}\right|=2 a_{p} .
$$

Its foci are at $\pm c_{p}$, and $a_{p}$ is the length of the major semi-axis. The normal to the surface at $x$ can thus be written as

$$
\nabla f_{p}=\frac{x-c_{p}}{\left|x-c_{p}\right|}+\frac{x+c_{p}}{\left|x+c_{p}\right|}
$$

That is, the normal bisects the lines joining $x$ to $\pm c_{p}$. As a consequence, all the normals 
(a)

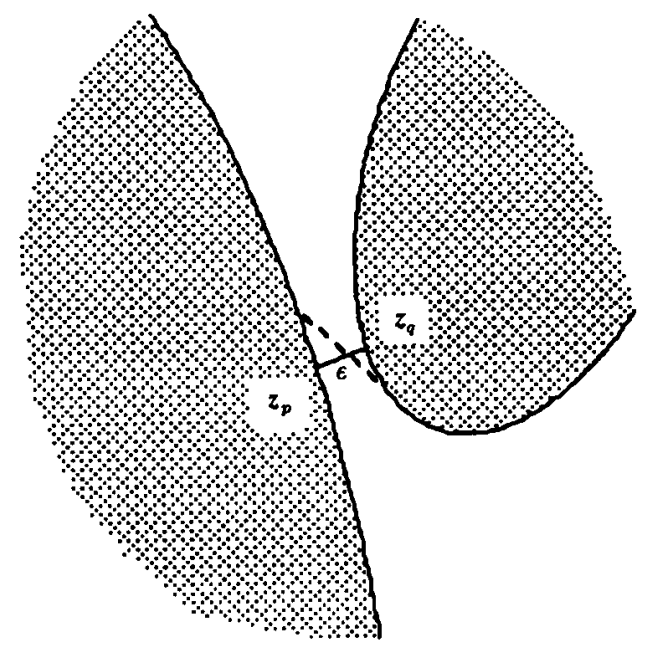

(b)

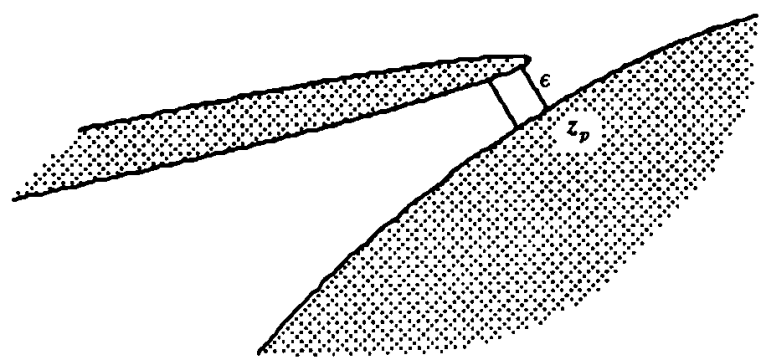

FIGURE 1. Sensitivity of the geometric features determining the magnitude of lubrication interactions to the location of the points of closest approach. (a) Although the points of closest approach are well approximated (on the scale of the particles), the estimate of the minimum separation $\epsilon$ is poor. (b) $\epsilon$ is estimated accurately, but the principal radii of curvature on the slender rod are incorrect.

to $A_{p}$ intersect the spheroidal axis between the foci. The normal common to $A_{p}$ and $A_{q}$ must therefore cross the symmetry axes of both particles, and $z_{p}$ and $z_{q}$ are the points where it penetrates their surfaces. The outline of our algorithm is thus as follows (figure 2):

(i) Find the intersection $\chi_{p}^{(k)}$ of the normal to $A_{p}$ at $z_{p}^{(k)}$ with the spheroidal axis. Repeat for particle $q$.

(ii) Find the intersections $z_{p}^{(k+1)}$ and $z_{q}^{(k+1)}$ of the line joining $\chi_{p}^{(k)}$ to $\chi_{q}^{(k)}$ with the surfaces $A_{p}$ and $A_{q}$.

(iii) Convergence check. (For instance, are the normals at $z_{p}^{(k+1)}$ and at $z_{q}^{(k+1)}$ nearly antiparallel?) Iterate (i)-(iii) if necessary.

The first guesses $z_{p}^{(0)}$ and $z_{q}^{(0)}$ must belong to the surfaces, but no other restrictions are placed on their accuracy, and one always finds $\lim _{k \rightarrow \infty} z_{p}^{(k)}=z_{p}$. This method has several advantages over alternatives. In the spherical limit, it corresponds to joining the centres of the particles, the simplest way to find $z_{p}$ and $z_{q}$ in that case. In general, we have reduced the dimensionality of the search domain from four (two curved boundaries) to two (two line segments). This obviously speeds up the convergence. Also, all schemes scanning the surfaces of the particles slow down dramatically as they near the points of closest approach, since the step size usually scales with $\left|z_{p}^{(k)}-z_{q}^{(k)}\right|$ in some fashion. In our method, on the other hand, the step size is set by $\left|\chi_{p}^{(k)}-\chi_{q}^{q(k)}\right|>\left|z_{p}^{(k)}-z_{q}^{(k)}\right|$. Finally, this algorithm is guaranteed to have a real solution at 


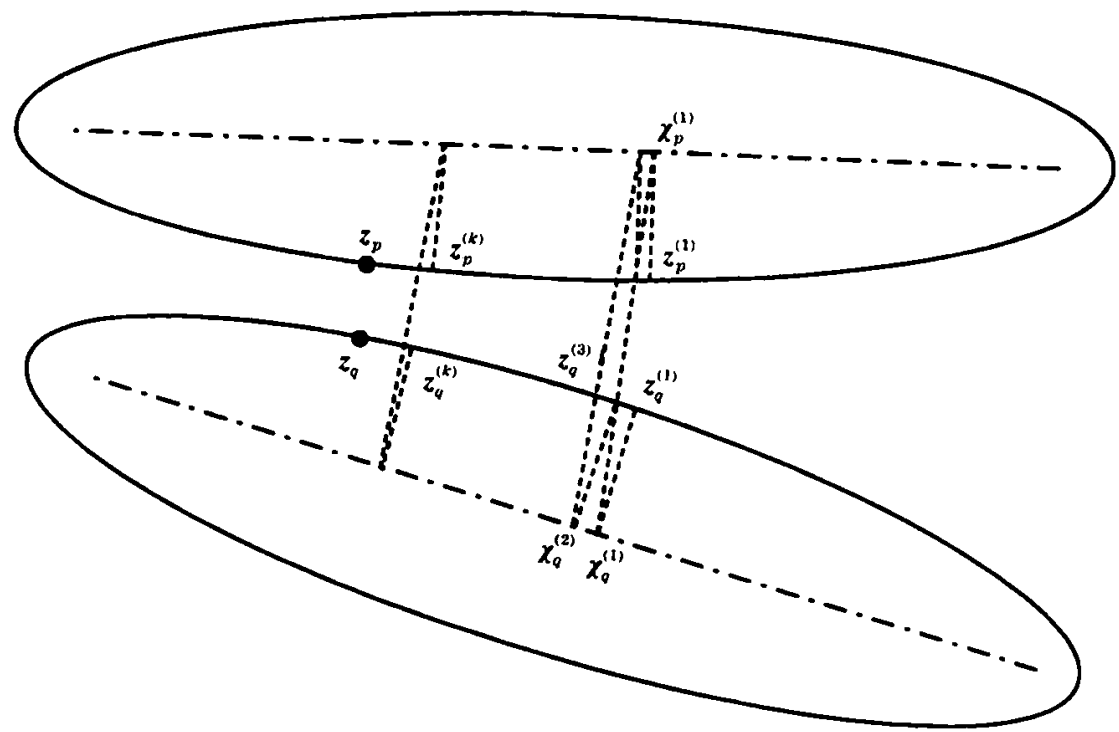

FigURE 2. Illustration of the algorithm to find the points of closest approach on two prolate spheroids.

each iteration. By contrast, many other methods which 'shoot' from one surface to the other, may occasionally 'miss', especially if the first estimates were poor, or if one of the points of closest approach is very near the tip of a slender ellipsoid. We note that this method also detects the points of maximum penetration in the case of overlapping spheroids (as long as the line segment $\left[x_{p}-c_{p}, x_{p}+c_{p}\right]$ is exterior to $A_{q}$ ), and that its concept can be extended to arbitrary ellipsoids (in which case $\chi_{p}^{(k)}$ belongs to the elliptic disk confocal with $A_{p}$ ).

The local geometry near the points of closest approach may readily be found by expanding $f_{p}$ (see (4.1)) in a Taylor series about $z_{p}$. We then apply the lubrication formulae (Claeys \& Brady 1989) to build the resistance tensor to $O(1)$ for each pair of particles near contact (i.e. for each set of spheroids for which the surface-to-surface separation, normalized by the harmonic mean of the local radii of curvature, is smaller than a prescribed threshold). This information is then added to $\boldsymbol{M}^{-1}$ to obtain $\boldsymbol{R}$ (see (2.7)). At this level of accuracy, the correction term $\mathbf{R}_{\text {corr }}$ is irrelevant, since it would only contribute to the unknown $O\left(\epsilon^{0}\right)$ part of the lubrication stresses.

\section{Selected examples of simulations for a finite number of prolate spheroids interacting hydrodynamically in a viscous fluid}

\subsection{Numerical aspects}

Using the method outlined in the preceding sections, we carried out a variety of simulations for systems involving a finite number of prolate spheroids in an unbounded Newtonian incompressible fluid. Because of numerical rather than conceptual limitations, we restricted the range of aspect ratios from $1+2 \times 10^{-6}$ to about 50 . The lower bound is so close to the spherical case that it can hardly be called a limitation. It springs from difficulties in computing the coefficients $\alpha$ and $\gamma$ in the expressions for the self-terms $M^{p p}$ (see (3.6) and Appendix B), and could easily be removed. The most efficient way of dealing with spheres, however, is to take their shape explicitly into account, and replace the line distribution of singularities used here (even though the 


$\begin{array}{cccl}r_{p} & \left|x_{p}-x_{q}\right| /(2 a) & |F| /(8 \pi a \mu|U|) & |\boldsymbol{F}| /\left|F_{\text {rel }}\right| \\ 2 & 1.0001 & 0.31483 & 0.9945 \\ 2 & 2 & 0.36788 & 0.9998 \\ 2 & 3 & 0.39216 & 1.000 \\ 2 & 16 & 0.43908 & 1.000 \\ 5 & 1.0001 & 0.20423 & 0.9938 \\ 5 & 2 & 0.23512 & 1.000 \\ 5 & 3 & 0.24546 & 1.000\end{array}$

TABLE 1. Drag on two prolate spheroids moving with equal velocity $U$ along their line of centres. The first column $\left(r_{p}\right)$ gives the aspect ratio of the particles. $\boldsymbol{F}_{\text {ret }}$ is the final result given with five significant digits in Gluckman et al. (1971). The smallest separation considered is 1.0001 instead of 1 because the resistance tensor is singular when the surfaces touch.

number of unknowns and speed up the computations. This was done in a few cases, but most simulations were performed using a general version of the code capable of handling fully three-dimensional particle conformations and polydisperse samples. The symmetry inherent to the method (mostly through Lorentz' reciprocal theorem) makes it very robust, and we never noticed any violations of the symmetry imposed by the initial conditions in any of our simulations.

Because of the strong coupling between the torque and the rate of strain $\left(\boldsymbol{M}_{T E}^{p q}\right.$ is non-zero even for $p=q$ ), it does not make sense for spheroids to consider an ' $\mathrm{F}$ - $\mathrm{T}$ 'method as proposed by Durlofsky et al. (1987). They found that useful results could be obtained for spheres (in the absence of extensional components in the impressed flow) by retaining only the couplings between the force and torque on the particles, and their translational and rotational velocities. They recognized, however, that they owed this success to the fact that all stresslets are induced in such circumstances. This is no longer true for ellipsoidal particles, because a single spheroid in a vorticity flow experiences strain. It is therefore necessary to keep the symmetric as well as the antisymmetric part of the first moment of the stress density on the particle surface, i.e. one must use the full ' $\mathrm{F}-\mathrm{T}-\mathrm{S}$ '-method of Durlofsky et al. (1987).

To test the accuracy of our method, we checked our results against those obtained by other researchers using various numerical techniques (vide infra). We also verified for a large number of multiparticle configurations that the grand resistance tensor that we calculate in the limit of zero eccentricity matches the one found using the version of Stokesian dynamics dedicated to spheres. All calculations were performed on a Sun $4 / 360$ workstation and typically required at most a few seconds of CPU time. The duration of dynamic simulations such as those presented in $\$ 5.4$ and 5.5 depends on the number of particles and their aspect ratio. Few of the examples given here however, were generated in more than 10 minutes. The program is written in FORTRAN, and its algorithm could readily be adapted for implementation on parallel computer architectures.

\subsection{Axisymmetric flow past chains of spheroids}

Gluckman et al. (1971) introduced the multipole collocation method to calculate the drag on equidistant identical spheroids, lined up in a queue, and moving with uniform velocity. We list in table 1 the force necessary for this motion in the case of two ellipsoids of aspect ratio 2 and 5 for various separations, and note excellent agreement with all published results. The accuracy of our results matches that achieved by Gluckman et al. using three or more 'multilobular disturbances' emanating from the centre of each particle, and is always within less than $1 \%$ of their converged results. 
line segment becomes infinitesimally short) by the appropriate combination of point forces, doublets and other multipoles at the sphere's centre (as done in Stokesian dynamics up till now (Durlofsky et al. 1987)). This is trivial to do. The upper bound $r_{p} \approx 50$ comes from more subtle numerical aspects. The major difficulty in the acicular limit is the smallness of the radii of curvature near the tip of the slender body. Since lubrication interactions only set in at surface separations small compared to that dimension, extremely close encounters between particles can occur before they experience any repulsion. The time step in dynamic simulations is mostly determined by the requirement that there would be (virtually) no particle overlap; when the superficial separations are so small, demands on the computer time become prohibitively large. This problem is by nature non-existent for static simulations (in which the evolution of the configuration is not tracked), so that one can then study fibres with much larger aspect ratios.

Particle paths are integrated using a fourth-order Adams-Bashford formula (Abramowitz \& Stegun 1970). The time step is chosen suitably small to make errors due to the time integration insignificant, its magnitude scaling with a characteristic velocity of the system and either the minimal separation between the particles or the smallest dimension of the spheroids. The mobility tensor was usually built and inverted for each new configuration, since far-field interactions dominate the dynamics of most applications involving only a few spheroids. For more concentrated systems, considerable savings in computer time may be achieved by updating the mobility tensor less frequently than the lubrication interactions. (The gross features of the $\mathrm{N}$ body configuration, which determine $M$, vary slowly on the timescale of the trajectory calculation, which is set by the requirement that typical displacements do not exceed the smallest gap width between particle surfaces.) Optimal use was made of Lorentz' reciprocal theorem for the construction of $M$. To minimize the number of required operations even further, we exploited the interesting identities (see Appendix C):

$$
\begin{aligned}
& \boldsymbol{M}_{U T}^{p q}=\frac{1}{2} \boldsymbol{\varepsilon} \cdot\left(\boldsymbol{\delta}: \boldsymbol{M}_{U S}^{p q}\right), \\
& {\left[\boldsymbol{M}_{\Omega T}^{p q}\right]_{i j}=-\left[\boldsymbol{M}_{E S]_{i k l j}^{p q}} \boldsymbol{\delta}_{k l}+\frac{1}{2}\left[M_{E S}^{p q}\right]_{m k l n} \delta_{k l} \delta_{m n} \delta_{i j},\right.} \\
& M_{\Omega S}^{p q}=\varepsilon \cdot M_{\Omega T}^{p q}+\left(\varepsilon \cdot M_{\Omega T}^{p q}\right)^{\mathrm{T}} .
\end{aligned}
$$

These hold for ellipsoids regardless of whether $p=q$. From these relations, it can be seen that only the couplings $M_{V F}, M_{U S}$ and $M_{E S}$ need to be evaluated for each pair of particles in order to build the grand mobility tensor $\boldsymbol{M}$. Taking maximal advantage of the tracelessness of $E$ and $S$, and of the symmetry of $M_{U F}$ (see (3.11)) and of $M_{E S}$ (Appendix $C$ ), this reduces the number of unknown independent mobility functions to 36 per pair of particles. (For the self-terms, only 21 are needed since $\boldsymbol{M}_{U S}^{p p}=0$ for spheroids.) The identities (5.1) also imply the following, previously apparently undiscovered relations between the coefficients $\alpha$ and $\gamma$ defined by Chwang $\& \mathrm{Wu}$ $(1974,1975)$ (see also Kim $1985 b$ and Appendix B):

$$
\begin{aligned}
\gamma^{-1} & =\frac{1}{2} \alpha_{5}^{-1}-\alpha_{4}^{-1}, \\
\gamma^{\prime-1}-\gamma^{-1} & =\frac{e^{2}}{2-e^{2}}\left(\alpha_{4}^{-1}-\frac{3}{2} \alpha_{5}^{-1}\right), \\
\alpha_{4}^{-1}-\frac{3}{2} \alpha_{5}^{-1} & =\frac{2}{\left(2-e^{2}\right) \alpha^{*}+e^{2} \alpha_{1}} .
\end{aligned}
$$

The symmetry elements in some of the illustrative examples given below (mirror symmetry about a plane, planar configuration, etc.) can also be used to reduce the 


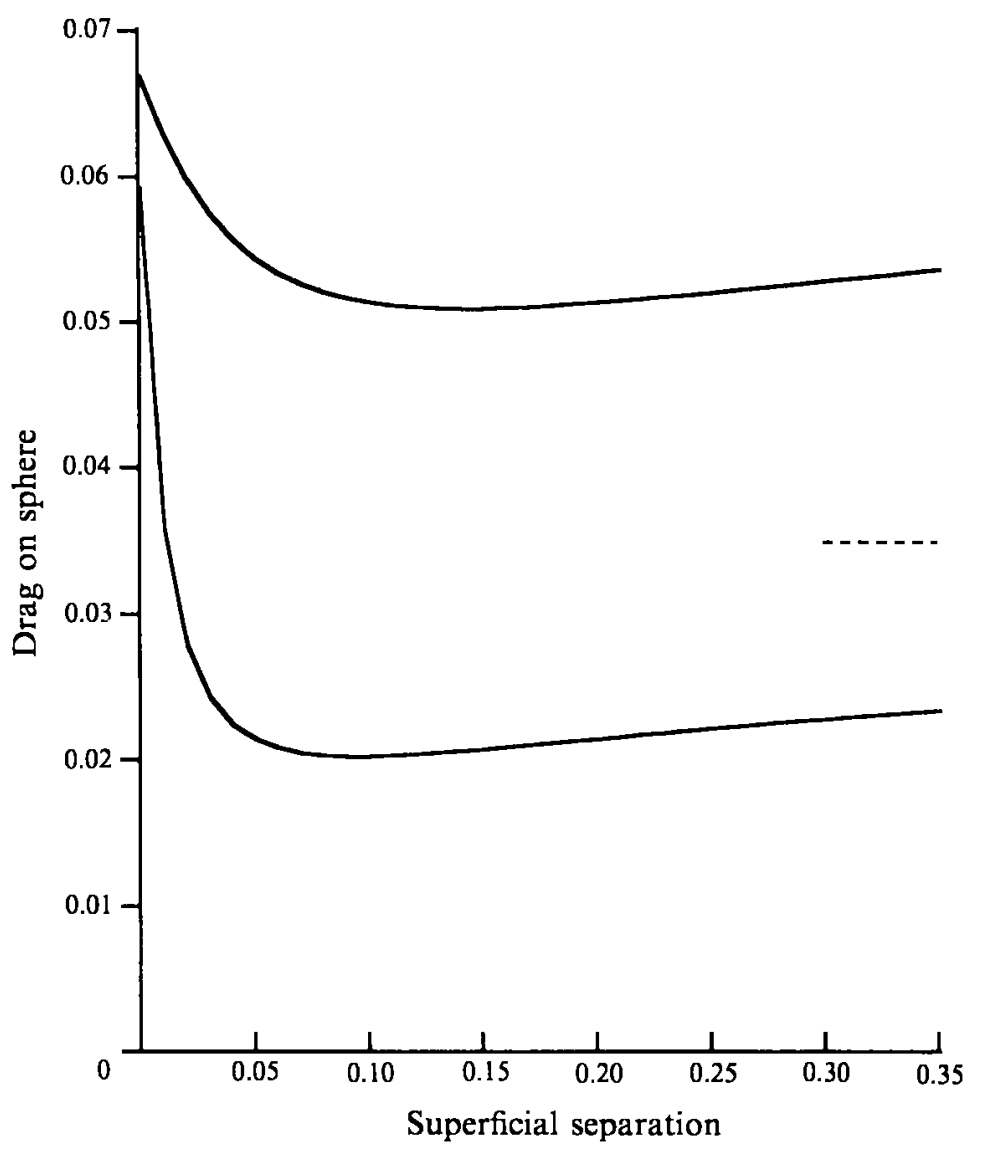

Figure 3. Drag on a small sphere placed at the tip of a spheroid of aspect ratio 10 moving with the same velocity $U$. Distance is non-dimensionalized by the half-length $a$ of the spheroid; the drag scales with $8 \pi a \mu U$. The bottom curve shows the drag for a sphere of radius 0.0464 ; the radius equals 0.1 in the other case. The force which would be experienced by the smallest sphere in the absence of the large particle is indicated by the dashed line. The asymptote for the sphere of radius 0.1 lies at 0.075 .

Our data for chains of up to 15 particles also are indistinguishable from their results (data not shown), except in the case of seven touching spheroids with $r_{p}=5$, where we calculated drags approximately $5 \%$ smaller than those deduced from their figure 11 .

The efficiency of the multipole collocation technique was later tested by Liao \& Krueger (1980) for ellipsoids of different size and aspect ratio. They calculated the force on a small sphere touching the nose of a large prolate spheroid of aspect ratio 10 as a function of the relative volume of the two bodies, which are assumed to move at the same speed in the direction of the spheroid's axis. As shown in figure 3, we find that the sphere experiences a minimal force when it is slightly ahead of the ellipsoid, rather than against it. This peculiar behaviour is reminiscent of the results of Cooley \& O'Neill (1969), who calculated the resistance of two unequal spheres moving at the same velocity along their lines of centres as a function of their separation (using the analytical solution for this problem due to Stimson \& Jeffery 1926). They found that, for sufficiently dissimilar radii, the drag on the large sphere first decreases with increasing distance between the particles, goes through a minimum and then monotonically ascends to its asymptotic value at large separations. It is possible that an analogous phenomenon occurs here. The harmonic mean radius of curvature at the 


\begin{tabular}{rcclcl}
$V_{1} / V_{2}$ & $R_{\mathrm{s}} / a$ & $\left|F_{1}\right|$ & \multicolumn{1}{c}{$\left|\boldsymbol{F}_{2}\right|$} & $\left|\boldsymbol{F}_{2}\right| /\left|\boldsymbol{F}_{\mathrm{s}}\right|$ & $r_{\mathrm{m}} / \boldsymbol{a}$ \\
1 & 0.2154 & 0.1565 & 0.1255 & 1.04 & 0.2 \\
10 & 0.1000 & 0.1766 & 0.050894 & 1.10 & 0.14 \\
100 & 0.0464 & 0.1880 & 0.020192 & 1.25 & 0.09 \\
1000 & 0.0215 & 0.1937 & 0.007779 & 1.38 & 0.06
\end{tabular}

TABLE 2. Drag on a small sphere preceding a spheroid of aspect ratio 10 moving at the same velocity. The spheroid and the sphere move with equal velocity $U$ in the direction of the rod's axis. The first two columns determine the relative size of the particles, as a ratio of volumes $\left(V_{1} / V_{2}\right.$, where the subscript ' 1 ' denotes the spheroid), or in terms of the radius of the sphere $R_{\mathrm{s}}$ relative to the major semi-axis $a$ of the spheroid. The forces $F_{1}$ and $F_{2}$ are scaled by $8 \pi a \mu U$. They are evaluated when the clearance between both bodies is $r_{\mathrm{m}}$, chosen such that the drag $F_{2}$ on the sphere is minimal. $F_{\mathrm{s}}$ is the value reported by Liao \& Krueger (1980) for the force on the sphere when both particles are touching.

pole of the spheroid is $a / r_{p}^{2}$. For a spheroid of unit half-length and aspect ratio 10 , this equals 0.01 , which is considerably smaller than the radius of the spheres considered in this analysis (see table 2). For reference, the volume of a sphere of radius $R_{\mathrm{s}}=0.01 a$ is only $10^{-4}$ times that of the ellipsoid. Thus it can be argued that, in a local sense, the spheroid looks like a smaller object than the sphere, strengthening the analogy with the work of Cooley \& O'Neill (1969). In table 2, we compare the calculated minimum drag on the sphere to the collocation results for touching particles (Liao \& Krueger 1980). Our computations systematically predict a higher force, and the agreement gets worse as the relative size of the two bodies departs more from unity. Liao \& Krueger remarked that the drag calculated using the multipole collocation technique monotonically approached a plateau value from below as the stick boundary condition was satisfied at more points on the large spheroid, but they fixed the number of collocation points on the small particle at four, relying on earlier studies for touching spheres. In some respects however, as pointed out above, the tip of the ellipsoid resembles an object smaller than the sphere presumed small in the analysis. Thus it may have been necessary to represent the sphere by more multipoles, and the truncation after the fourth term may have caused an underestimation of the true drag force. In the same light, it is certain that our method would be more accurate if contributions from the quadrupole, octupole and higher moments were retained.

\subsection{Drag on two acicular spheroids}

Instead of adopting the distribution of singularities suggested by the Faxén laws, Barta \& Liron (1988) determined the optimal density of Stokeslets along the symmetry axis of the spheroids as part of the solution procedure, by equating the disturbance velocity at the particle's surface to the imposed rigid-body motion. Since they collapse the surface stress density onto a line, yet neglect singularities more complex than point forces, they cannot fully capture effects arising from the finite thickness of the rods. In particular, it is uncertain that a distribution of Stokeslets confined between the foci of the spheroid can correctly represent the flow field in the immediate vicinity of the particle's surface, as they assume. For two parallel ellipsoids in an unbounded fluid, the drag was calculated at various separations for synchronous motions along the axes $\left(U_{1}=U_{2}\left\|d_{1}\right\| d_{2}\right)$, along the line of centres, and perpendicular to the plane defined by the directors of the particles. We repeated the analysis and obtained the results of table 3. They agree quite reasonably with the forces calculated by Barta \& Liron (1988). As was to be expected, the largest deviations occur in situations when hydrodynamic interactions are most pronounced, i.e. for motion in the direction of the line of centres, 


\begin{tabular}{ccllllll} 
& \multicolumn{2}{c}{$\left|F_{b}\right|$} & & \multicolumn{2}{c}{$\left|F_{n}\right|$} & \multicolumn{2}{c}{$\left|F_{d}\right|$} \\
\cline { 2 - 3 } 0.25 & 0.1168 & $(1.30)$ & 0.1300 & $(1.12)$ & 0.08009 & $(1.10)$ \\
1 & 0.1382 & $(1.07)$ & 0.1510 & $(1.021)$ & 0.09413 & $(1.012)$ \\
2 & 0.1497 & $(1.024)$ & 0.1597 & $(1.006)$ & 0.09885 & $(1.003)$ \\
4 & 0.1593 & $(1.007)$ & 0.1655 & $(1.002)$ & 0.10151 & $(1.001)$ \\
6 & 0.1632 & $(1.003)$ & 0.1677 & $(1.001)$ & 0.10240 & $(1.000)$
\end{tabular}

TABLE 3. Drag on two spheroids of aspect ratio 100 placed side by side. In all cases the velocities $U_{1}=U_{2}=U$. All forces have been non-dimensionalized by $8 \pi a \mu \mid U$. The distance $d$ is measured between the centres of the particles. $\left|F_{b}\right|$ is the drag for motion along the line of centres, $\left|F_{n}\right|$ for movement perpendicular to the plane in which the spheroidal axes lie, and $\left|F_{d}\right|$ is the force for $U\left\|d_{1}\right\| d_{2}$. The entries in parentheses give the ratio of the drag calculated by Stokesian dynamics to the data found in Barta \& Liron (1988).

and at the smallest separations. In those cases, the optimal Stokeslet distribution calculated by Barta \& Liron deviated significantly from the uniform density profile predicted for isolated particles by Chwang \& Wu $(1974,1975)$, but by about $20 \%$ at most. It is worth mentioning that Barta \& Liron must compute a new singularity distribution for each prescribed motion, while Stokesian dynamics solves all resistance problems simultaneously for a given geometry. Our method also adequately captures the small rotation induced by the motion of the spheroids parallel to their axes (data not shown).

\subsection{Sedimentation of spheroids}

The motion of two spheroids sedimenting side by side is intriguing since 'periodic' orbits appear for certain initial conditions (Kim 1985a). Hydrodynamic interactions then cause the particles' directors to rotate past $\frac{1}{2} \pi$ radians, at which point the trajectory - projected onto a plane perpendicular to gravity - is reversed (figure $4 b$ ). At larger separations, the viscous interactions are weaker, and the bodies simply drift apart (figure $4 a$ ). Similar effects had been observed for highly symmetric arrangements of spheres (Durlofsky et al. 1987); four spheres placed at the corners of a square in the vertical plane, for example, fall in a viscous fluid following a pattern in which the top spheres first move inward and faster than the ones on the bottom, eventually overtaking these to form a new square which is the mirror image of the original configuration. This scenario is repeated ad infinitum in the absence of external perturbations.

One can generate many 'repeating' configurations of spheroids by positioning the particles' centres at the corners of a regular polygon at right angles with gravity $g$, with their directors all parallel to $g$ (or all at the same angle with gravity, such that the spheroidal axes lie on the envelope of a circular cone). The periodic trajectories described by the ellipsoids in the $((R / a), \theta)$-plane are shown for a few collections of spheroids of aspect ratio 2 in figure 5 . ( $R$ is the centre-to-centre distance between nearest neighbours and $\theta$ the azimuthal angle.) The time $t_{\mathrm{P}}$ necessary for the original configuration to be reproduced decreases at first with the number of spheroids in the system, as expected because of the more numerous hydrodynamic interactions (table 4). However, as the arrangement becomes more circular, the effects of the two nearest neighbours balance each other progressively more (the induced torques are nearly antiparallel) and the distance between spheroids diametrically opposed grows, weakening their interaction. As a result, we observe a minimum in $t_{\mathrm{p}}$ at $N=5$ for spheroids released at the corners of regular polygons of side $2 a$. If we fix the diameter of the polygon instead, we still observe a minimum $\left(t_{\mathrm{p}}^{\prime}\right.$, table 4$)$, now due to the strong 


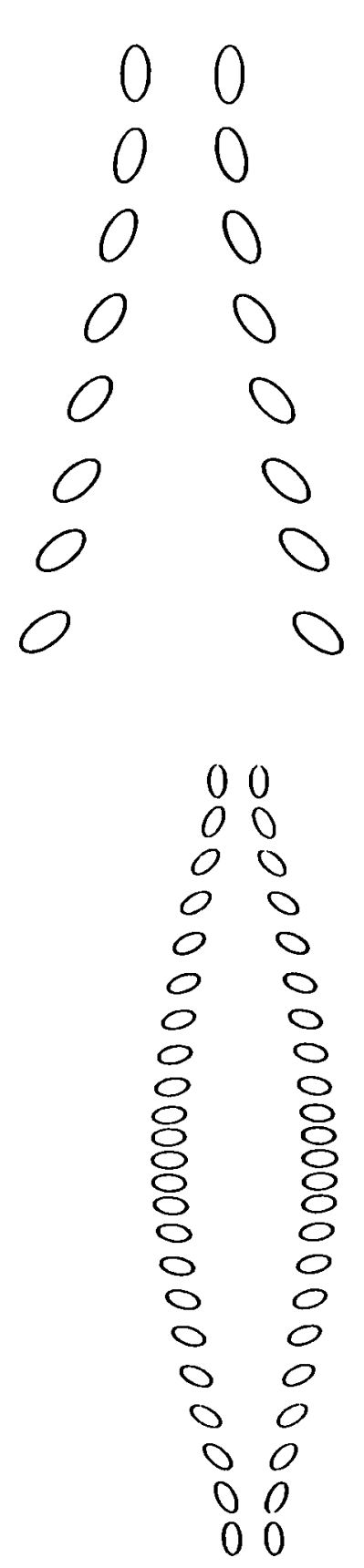

(a)

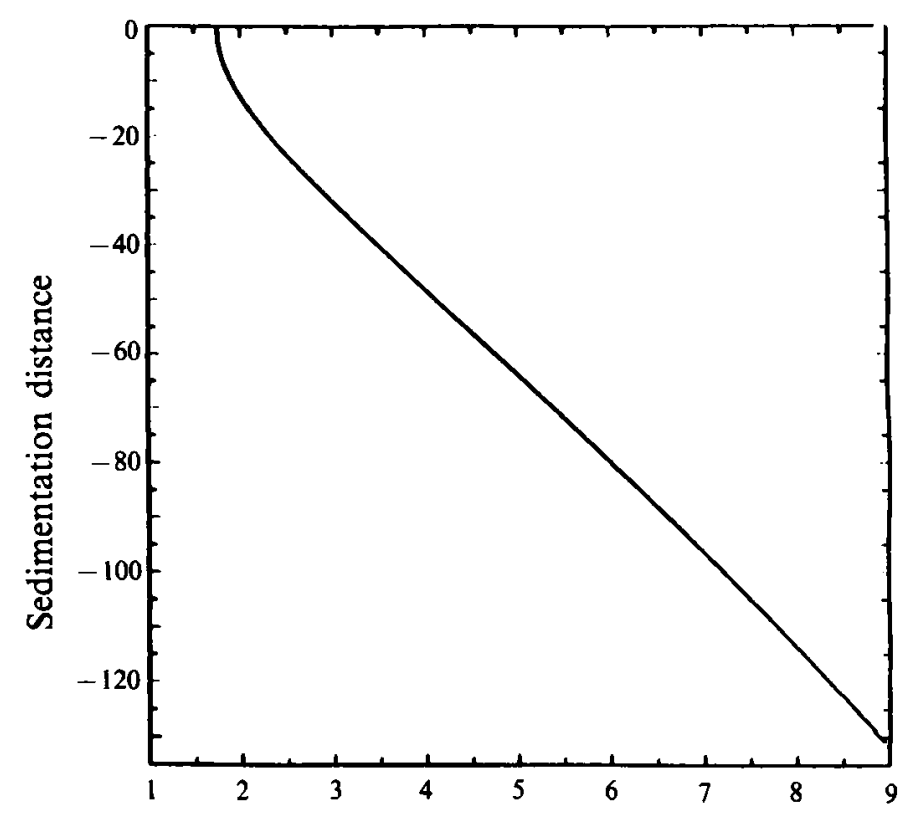

(b)

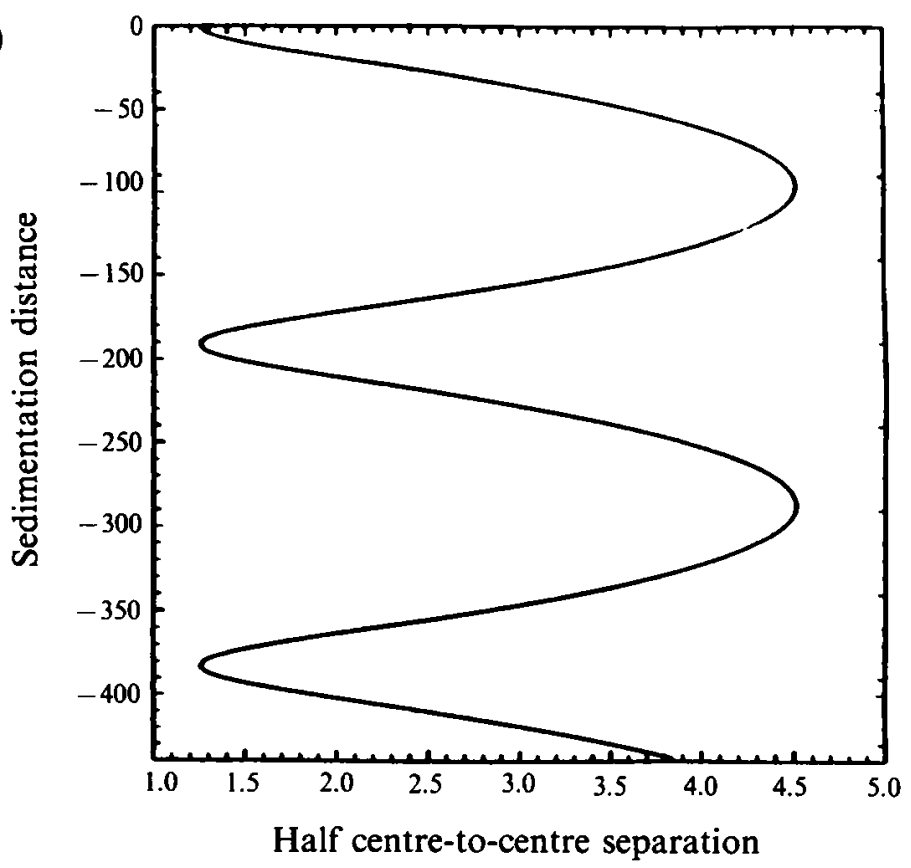

FIGURE 4. Sedimentation of two prolate spheroids of aspect ratio 2 at an initial centre-to-centre separation of $(a) 3.5$ and $(b) 2.5$ (non-dimensionalized by the length of the major semi-axis). The particles are released side by side and aligned with the direction of gravity. The sketch on the left represents snapshots of the ellipsoids taken at equal time intervals. The vertical distance has been scaled down in this illustration. 


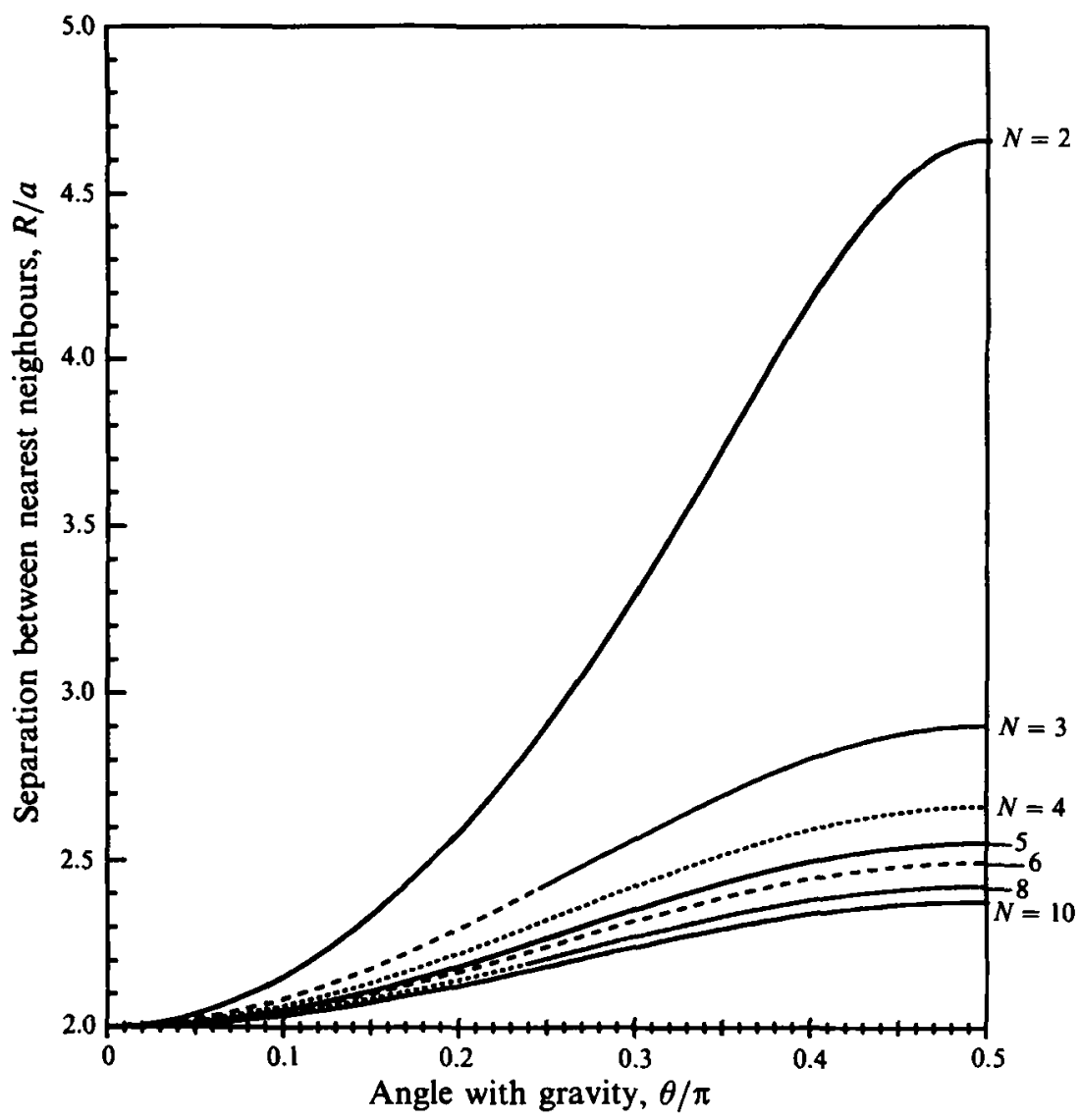

FIGURE 5. Periodic centre-of-mass trajectories of spheroids with aspect ratio 2 sedimenting at the corners of regular polygons of side $2 a$. The spheroids, with half-length $a$, are initially aligned with gravity $(\theta=0)$. All curves are symmetric about $\theta=\frac{1}{2} \pi$.

$\begin{array}{rcccc}N & t_{\mathrm{P}} & z\left(t_{\mathrm{p}}\right) & t_{\mathrm{p}}^{\prime} & z\left(t_{\mathrm{p}}^{\prime}\right) \\ 2 & 32.4 & 76.1 & 33.6 & 78.7 \\ 3 & 11.3 & 33.2 & 8.48 & 26.5 \\ 4 & 9.51 & 31.4 & 5.13 & 20.1 \\ 5 & 9.24 & 32.9 & 3.88 & 18.5 \\ 6 & 9.40 & 35.3 & 5.95 & 32.6 \\ 8 & 10.1 & 41.1 & & \\ 10 & 11.1 & 47.5 & & \end{array}$

TABLE 4. Period of similitude of highly symmetric configurations of sedimenting spheroids. This table reports some characteristics of the trajectories of $N$ identical sedimenting spheroids released parallel to gravity at the corners of regular polygons of side $2 a$ (columns $t_{\mathrm{p}}$ and $z$ ) or of diameter $2.02 a$ (columns $t_{\mathrm{p}}^{\prime}$ and $z^{\prime}$ ). The period of similitude $t_{\mathrm{p}}\left(\right.$ or $\left.t_{\mathrm{p}}^{\prime}\right)$ is defined as the minimum time elapsed between two instants at which the configuration of the spheroids is the same, except for a uniform translation by $z\left(t_{\mathrm{p}}\right)$ along the direction of gravity. (A spheroid pointing 'up' is considered equivalent to one pointing 'down'. If the sense of the director needs to be distinguished, the reported values of $t_{\mathrm{P}}$ and $t_{\mathrm{p}}^{\prime}$ should be doubled, since the configuration first reverses before reassembling.) Time is nondimensionalized by $|F| /\left(8 \pi \mu a^{2}\right)$, with $F$ the force of gravity. The distance $z$ travelled by the centre of mass of the arrangement is scaled by the particle's half-length $a$. 


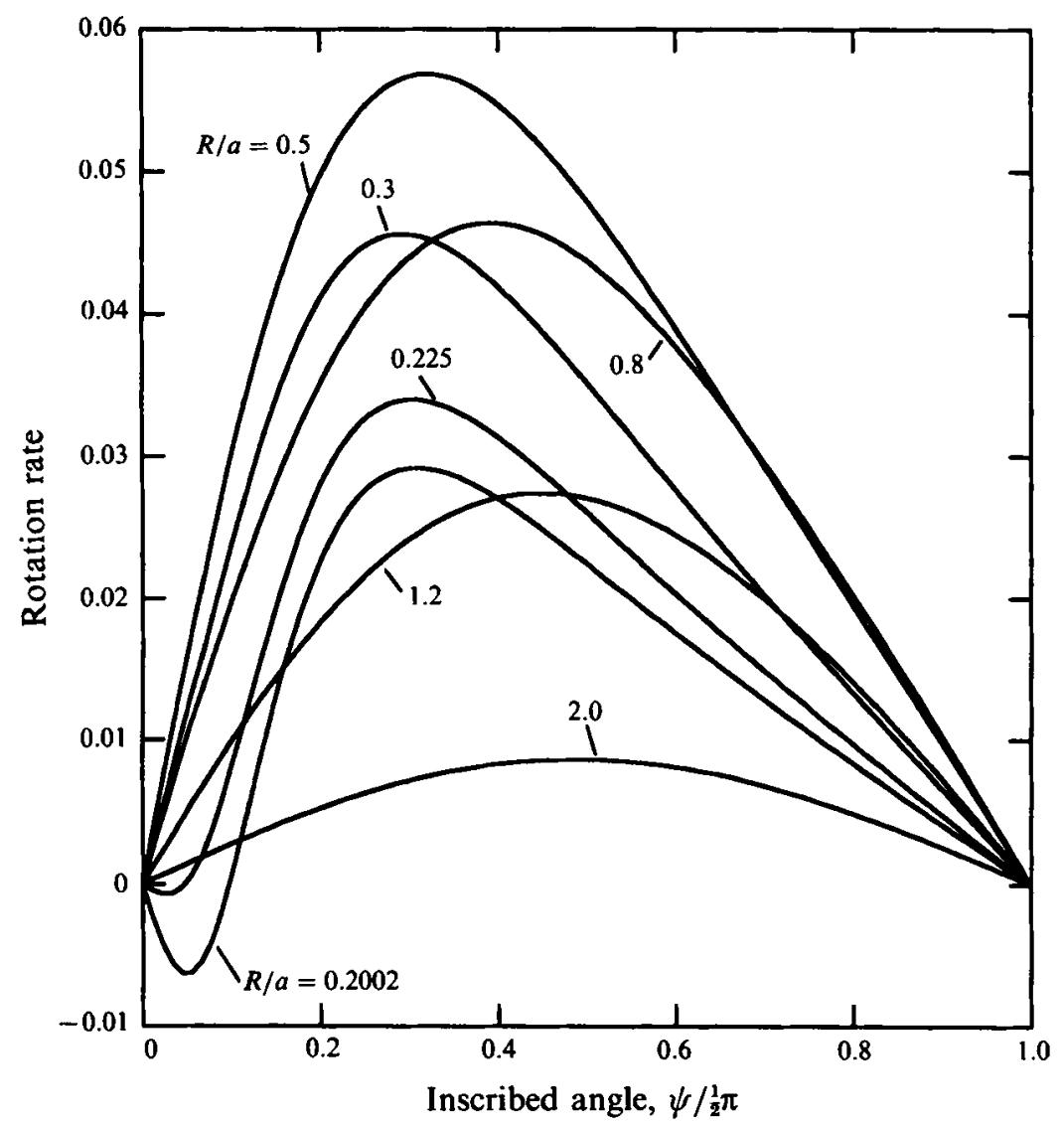

FIGURE 6. Rate of rotation of two spheroids of aspect ratio 10 sedimenting atop one another. The distance $R / a$ is the centre-to-centre separation. The speed of gyration is non-dimensionalized by $8 \pi a^{2} \mu /\left|F_{p}\right|$, with $F_{g}$ the force of gravity.

mutual hindrance of the tumbling spheroids as they are nearly closed packed. It is coincidental that both minima occur for pentagons. Note that, even though the period of similitude $t_{\mathrm{P}}$ is shorter for $N=5$ than for $N=4$, the particles travel farther in one cycle when they are arranged in a pentagon. This is due, of course, to the fact that the drag decreases with $N$, causing them to fall faster.

Two horizontal spheroids placed directly atop one another, but not perfectly aligned, spin while sedimenting because, as a pair, they have a propeller-like geometry. Our simulations show that both particles rotate in the same sense and at the same rate as they fall; there is no relative motion between the two bodies. (This behaviour is the only one consistent with the symmetries which the system must obey upon reversal of the direction of gravity.) The rate of rotation of the particles for different centre-tocentre separations $R$ is plotted as a function of the angle $\psi$ inscribed between the spheroidal axes in figure 6. Similar plots for other aspect ratios display the same qualitative features, but the magnitude of the speed of gyration is lower for blunter spheroids, and the maximum in the curves shifts toward the middle $\left(\psi=\frac{1}{4} \pi\right)$. The angle $\psi_{\mathrm{m}}(R / a)$ at which the tumbling is most rapid does not increase monotonically with separation $R$, but is minimal at about $\left\{R=0.3 a, \psi_{\mathrm{m}}=0.28 \pi / 2\right\}$ for rods of aspect ratio 10. For all aspect ratios considered, we found $\lim _{R \rightarrow \infty} \psi_{m}=\frac{1}{4} \pi$. This limit is always 
approached from below. The zero rotation rate for $\psi=\frac{1}{2} \pi$ can be proven from symmetry arguments.

The (small) negative rotation rate for almost touching particles and very acute inscribed angles can be explained by the trade-off between two competing effects. Those are best brought to light by considering the equivalent resistance problem and decomposing the motion of the spheroids: in the first case, the top spheroid is moved downward, holding the other particle fixed; in the second, the lower rod is pulled away from the stationary upper rod. We then superpose both motions to reproduce the original situation. In the first problem, a torque must be exerted on the moving spheroid to oppose its tendency to rotate at right angles to the lower rod. This can be understood by visualizing the fluid as being squeezed more tightly in the sharp corner formed by the rods, than in the obtuse angle complementary to it. In the second case, the moving lower rod attempts to drag the upper rod in its wake; thus holding its stationary requires a torque in the opposite sense. The first effect is only important at very close separations, and for angles relatively far removed from $\frac{1}{2} \pi$, and explains the negative rotation rate in figure 6 . In most situations, the second contribution is the largest, and the propeller-like motion is observed. $\dagger$ As one would expect, this phenomenon is absent (or not noticeable) for spheroids of aspect ratio 2, and confined to a much smaller range of $\psi$ when $r_{p}=50$.

\subsection{Perturbation of Jeffery orbits and migration in shear flow}

A force-free isolated spheroid placed at the origin of an unbounded simple shear flow precesses about the vorticity axis with a period of $2 \pi\left(r_{p}+r_{p}^{-1}\right) / E$ (with $E$ the magnitude of the velocity gradient), but the particle's centre does not move (Jeffery 1922). The rate of rotation is not uniform however, and the particle spends most of the time aligned with the flow. This tumbling motion suggests that the stress response of a dilute suspension of rods in simple shear is oscillatory. Experimental evidence abounds however (Ivanov, van de Ven \& Mason 1982), indicating that these fluctuations are transient, and that a well-defined, time-independent macroscopic viscosity can eventually be assigned to the dispersion. Many randomizing factors have been implicated, including polydispersity and other imperfections in the particle shape, Brownian motion, and hydrodynamic interactions (including wall effects). It is therefore instructive to examine the motion of pairs of non-Brownian, identical spheroids in shear flow to isolate the role of viscous forces.

The geometry considered consists of two ellipsoids of aspect ratio 2 which are mirror images of each other with respect to the plane of shear (figure 7). $\ddagger$ They are inclined at an angle $\frac{1}{3} \pi$ relative to the vorticity axis, and are initially either perpendicular to the velocity gradient $\left(\left.\phi\right|_{t=0}=0\right)$, or to the direction of flow $\left(\left.\phi\right|_{t=0}=\frac{1}{2} \pi\right)$. Somewhat surprisingly, the particles migrate due to hydrodynamic interactions, even though the undisturbed fluid velocity at their centres is zero. When the particles start off aligned with the flow $\left(\left.\phi\right|_{t=0}=0\right)$, they cycle on a closed trajectory, moving atop one another in a fashion similar to the jaws of a nutcracker. The composite centre of mass follows an 8-shaped loop in the plane of shear in the clockwise direction (figure 8); the rods, meanwhile, 'open' and 'close', making the most acute angle with the vorticity axis when they reach the top and the bottom of the 'figure of 8 ' shape. This motion can be

$\dagger$ We are not aware of any experiments confirming the reversal of the sense of rotation predicted here. However, the balance between the opposing effects, and hence this phenomenon, is somewhat sensitive to the exact shape of the rods. We were unable to reproduce it numerically, for instance, using strings of spherical beads instead of spheroids.

$\ddagger$ This problem was first suggested in a personal communication by B. J. Yoon \& S. Kim. 


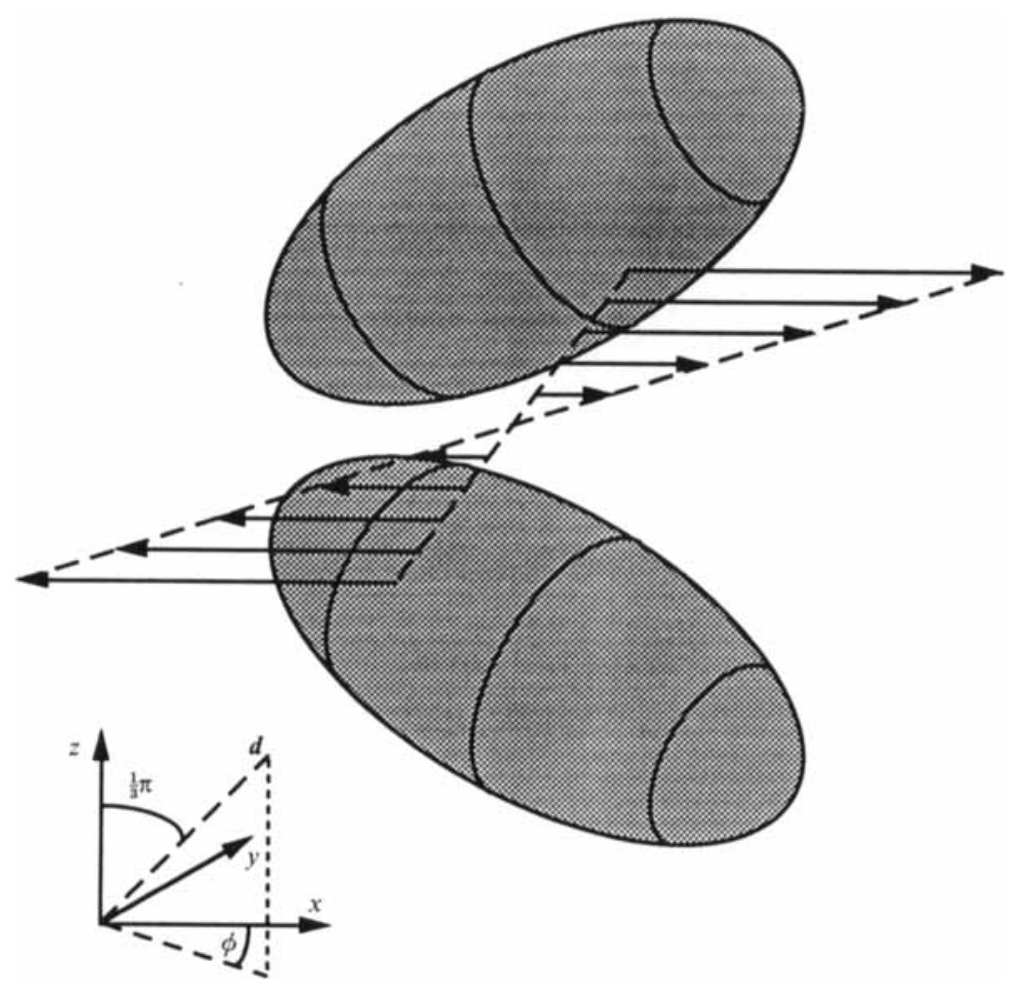

FIGURE 7. Geometry considered in $\S 5.5$. The vector $d$ indicates the orientation of the particles. $z$ lies along the vorticity of the shear field, and $x$ is the direction of the flow. The angle $\phi$ is measured in the $(x, y)$-plane.

rationalized more easily perhaps by picturing dumbbells instead of spheroids. Initially, the simple shear exerts a torque on the particles, causing them to spin. It experiences less resistance from the ends of the rods which are close together (the hinge of the nutcracker) however, than from the poles which are farther apart. (In the same manner, the combined drag on two nearly touching spheres is less than twice the force felt by an isolated particle.) The effect of this imbalance is a net displacement of the rods in the same direction as the 'hinge'.

If the spheroids are initially at right angles to the fluid velocity, their interactions result in a finite translation in the flow direction during each period of rotation. At the inflexion point in the orbit of the centre of mass (figure 8), the particles are perpendicular to the velocity gradient $(\phi=0)$ and the inscribed angle between their axes is minimal. Note that the behaviour is very similar to the former case, $\left.\phi\right|_{t=0}=0$, except that, when $\phi=0$ here, the centroid lies on a streamline with non-zero velocity. Lubrication interactions are, on average, less important in the second case, and the motion perpendicular to the plane of shear is consequently much less pronounced. The period of rotation is 15.66 for rods initially at right angles to the flow and 15.53 when $\left.\phi\right|_{t-0}=0$. (Time is non-dimensionalized by the magnitude of the velocity gradient.) For comparison, it is 15.71 for an isolated spheroid of aspect ratio 2 . The slight decrease can be understood since the pair of ellipsoids effectively acts as a body with a lower aspect ratio. 


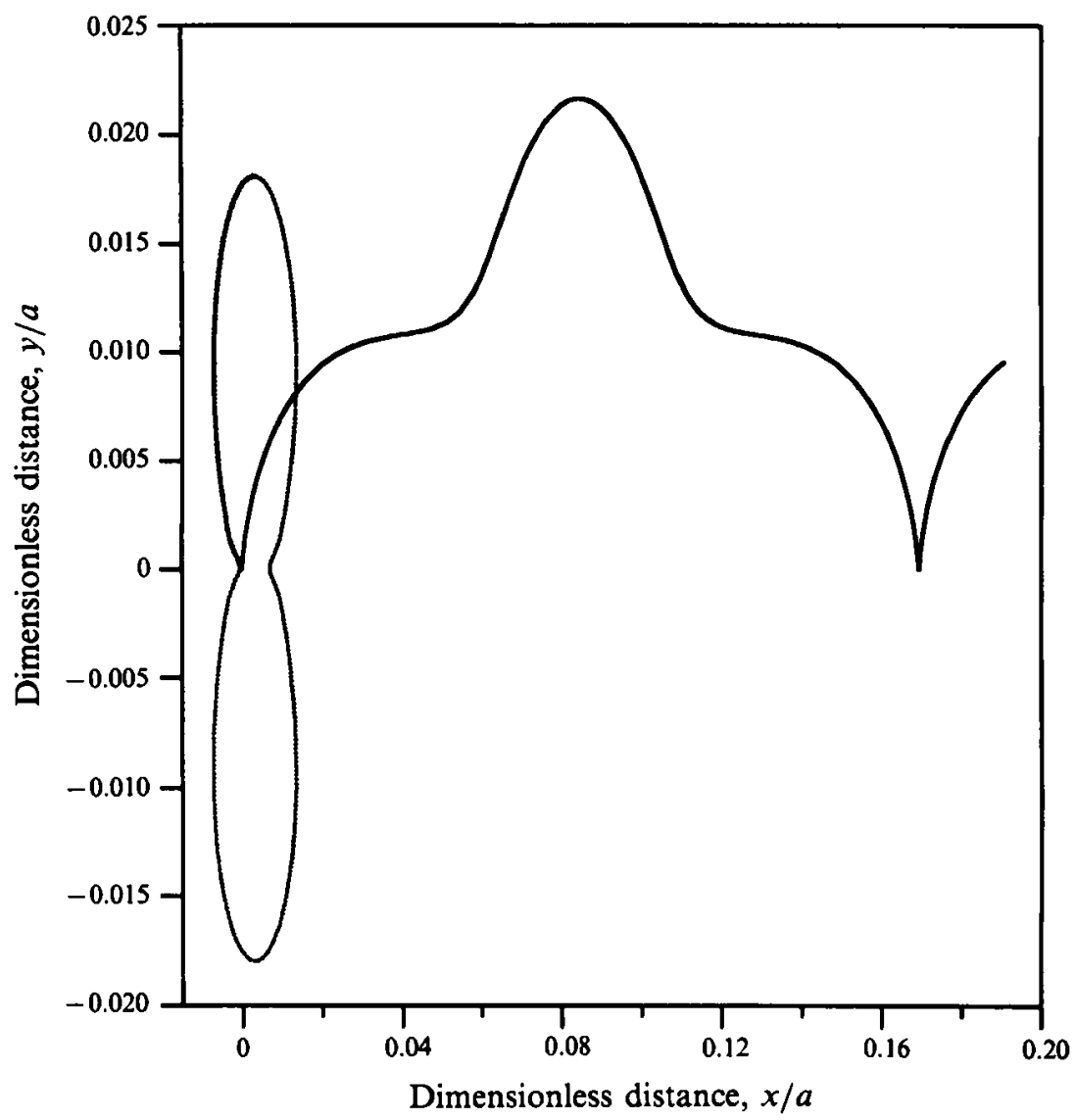

FIGURE 8. Shear-induced migration of two spheroids of aspect ratio 2 . The abscissa, $x / a$, denotes a displacement in the direction of the undisturbed streamlines (figure 7). The ordinate, $y / a$, measures distances travelled in the plane of shear, perpendicular to the flow. The closed orbit (dotted line) is described if $\phi=0$ initially. For $\phi=\frac{1}{2} \pi$, the open trajectory (solid line) is followed.

\section{Concluding remarks}

The examples of the preceding section demonstrate the effectiveness and accuracy of the moment expansion technique that we have developed for Stokes flow problems involving interacting spheroids. Because it is analogous in spirit to the established simulation method for spheres (Brady \& Bossis 1988), we also use the name 'Stokesian dynamics' to describe this new technique. In fact, the fundamental concepts of Stokesian dynamics can be stated in very general terms (Claeys 1991), and its methodology is valuable for a much wider class of body geometries than ellipsoids only. We discussed its application to prolate spheroids in particular in $\$ 3$ and 4 , and have shown that our approach compares quite favourably with other numerical methods.

Although many relevant problems can be addressed by considering the interactions between only a few particles, the usefulness of this new technique would be much increased if it were applicable to systems containing a very large (infinite) number of solid bodies. Most importantly, this would make it a valuable tool in suspension rheology, and enable one to study the flow properties of slurries of needle-shaped particles, the permeability of fibrous media, or the diffusion coefficients within liquid 
crystalline domains for instance. The extension of the method to unbounded dispersions constitutes the topic of our next article (Claeys \& Brady 1993a).

\section{Appendix A. Irreducible expansion for the disturbance velocity of an ellipsoid in Stokes flow}

We define the surface $A$ of an arbitrary ellipsoid by specifying that

$$
x \in A \Leftrightarrow A^{-1}: x x=1,
$$

with $\boldsymbol{A}$ any positive-definite symmetric second-rank tensor. Without loss of generality, we have set the origin $x=0$ at the geometric centre of the particle. It is apparent from (A 1) and the definition of the multipole moments

$$
\boldsymbol{P}^{(m)}=-\frac{1}{m !} \int_{A} n \cdot \sigma \stackrel{\boldsymbol{x}^{m}}{x} \mathrm{~d} A_{x}
$$

that any contraction of $\boldsymbol{P}^{(m)}$ by $\boldsymbol{A}^{-1}$ in any two of its last $m$ indices yields $\boldsymbol{P}^{(m-2)}$ (apart from a multiplicative constant). Resorting to index notation (with implicit summation over repeated indices),

$$
\left[\boldsymbol{P}^{(m)}\right]_{i_{0} i_{1} i_{2} \ldots i_{m}}\left[\boldsymbol{A}^{-1}\right]_{i_{k} i_{l}}=\frac{1}{m(m-1)}\left[\boldsymbol{P}^{(m-2)}\right]_{i_{0} i_{1} i_{1} j_{2} \ldots i_{m-2}}
$$

with $1 \leqslant k<l \leqslant m$ and $\forall p \in[1, m-2]:\left\{j_{p-1}<j_{p}\right.$ and $\left.k \neq j_{p} \neq l\right\}$.

We therefore define the irreducible moment $I^{(m)}$ for an ellipsoid such that all similar contractions give zero:

For instance,

$$
I^{(m)}=-\frac{1}{m !} \int_{A} n \cdot \sigma \stackrel{\times m}{x} \mathrm{~d} A_{x}
$$

$$
\begin{aligned}
& I^{(0)}=P^{(0)}=F \\
& I^{(1)}=P^{(1)}=S-\frac{1}{2} \varepsilon \cdot T+\frac{1}{3} P^{(1)}: \delta \delta \\
& I^{(2)}=-\frac{1}{2} \int_{A} n \cdot \sigma\left[x x-\frac{1}{3} A\right] \mathrm{d} A_{x}=P^{(2)}-\frac{1}{6} P^{(0)} A .
\end{aligned}
$$

In (A $4 c$ ), the first moment is broken down into its antisymmetric component, namely the torque $T=\varepsilon: P^{(1)}$, and a traceless symmetric part identified as the stresslet.

We now prove by induction that

$$
\overbrace{\boldsymbol{x}}^{\times m}=\sum_{j=0}^{|m / 2|} \frac{(2 m-4 j+1) ! !}{(2 m-2 j+1) ! !} \mathfrak{p}(\overbrace{\boldsymbol{x}}^{\times(m-2 j)} \overbrace{\boldsymbol{A}}^{\times j})
$$

or, equivalently, for $m>1$,

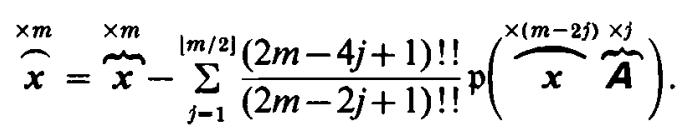

The notation $\lfloor x\rfloor \underset{\times m}{\text { means }}$ the largest integer value smaller than $\underset{x m}{x}$ or equal to $x$. As can be

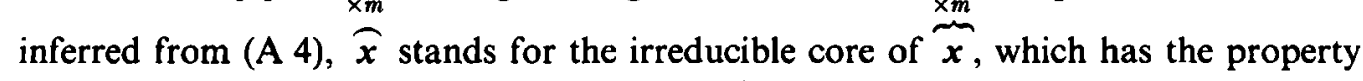
that all possible contractions by the dyadic $\boldsymbol{A}^{-1}$ give zero. (Alternatively, (A $5 b$ ) can be 
seen as its definition, in which case we must prove its irreducibility with respect to contractions by $\boldsymbol{A}^{-1}$.) The operation $\mathfrak{p}(\boldsymbol{X})$, with $\boldsymbol{X}$ a tensor of rank $m$, sums all distinguishable tensors which can be constructed from $\boldsymbol{X}$ by permuting its indices. For instance,

$$
\mathfrak{p}(x x y y)=x x y y+x y x y+x y y x+y x x y+y x y x+y y x x .
$$

It is an easy combinatorial problem to show that $(2 j+k) ! /\left(k ! 2^{j} j !\right)$ different permutations of the indices of $\overbrace{\boldsymbol{x}}^{\times x_{\boldsymbol{A}}^{\times j}}$ exist. Of these,

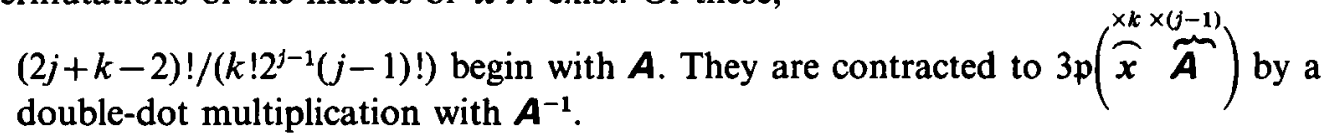

$(2 j+k-2) ! /\left(k ! 2^{j-2}(j-2)\right.$ !) begin with two indices belonging to different $\boldsymbol{A}$, like $[\boldsymbol{X}]_{i p q l}=[\boldsymbol{A}]_{i q}[\boldsymbol{A}]_{p l}$. These permutations yield $2(j-1) \mathrm{p}(\stackrel{\times \boldsymbol{x} \times(j-1)}{\boldsymbol{A}})$.

$\left(2(2 j+k-2) ! /\left((k-1) ! 2^{j-1}(j-1) !\right)\right.$ have the same first two indices as either $x A$ or $\boldsymbol{A} \boldsymbol{x}^{\mathrm{T}}$, and condense onto $2 k p(\stackrel{x \kappa \times(j-1)}{\boldsymbol{x}} \stackrel{\boldsymbol{A}}{\boldsymbol{A}})$.

$(2 j+k-2) !\left((k-2) ! 2^{j} j !\right)$ begin with the first two indices of $\frac{x_{x}}{x}$ and are contracted to zero by definition.

Therefore,

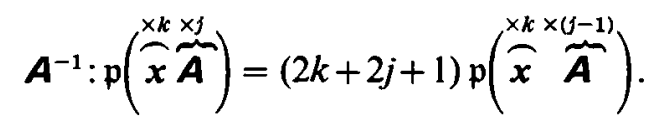

We now condense both sides of the relation (A $5 b$ ) with $A^{-1}$, and use $A^{-1}: x x=1$ to get

$$
A^{-1}: \stackrel{\times m}{\boldsymbol{x}}=\overbrace{\boldsymbol{x}}^{\times(m-2)}-\sum_{j=1}^{|m / 2|} \frac{(2 m-4 j+1) ! !}{(2 m-2 j-1) ! !} \mathfrak{p}(\overbrace{\boldsymbol{x}}^{\times(m-2 J) \times(j-1)} \overbrace{\boldsymbol{A}}),
$$

which is zero by induction from (A $5 a$ ) (QED). Obviously, the proof does not depend on which two indices of $\overbrace{x}^{x}$ are condensed, and all multiple contractions of $\overbrace{x}^{x}$ by permutations of $\overbrace{A^{-1}}^{x_{j}}$ (with $0<j \leqslant[m / 2]$ ) will also yield zero. Finally, it is trivial to show that the premise (A $5 a$ ) holds for $m=0$ and $m=1$, since $\overbrace{x}^{x_{0}}=1$ and $\overbrace{x}^{x}=x$, which are both irreducible. This completes the proof of (A $5 a$ ).

We now prove the following proposition for any sufficiently smooth tensorial function $\boldsymbol{X}(\boldsymbol{x})$ :

$$
X(x)=\left.\sum_{m=0}^{\infty} \frac{(2 m+1) ! !}{m !} \overbrace{\boldsymbol{x}}^{x_{m}} \odot^{m}\left[\left(\frac{1}{\mathrm{D}} \frac{\mathrm{d}}{\mathrm{dD}}\right)^{m} \frac{\sinh \mathrm{D}}{\mathrm{D}}\right] \widetilde{\nabla}^{x^{m}} X(y)\right|_{y=0} .
$$

We begin with the Taylor series expansion for $X$ about $x=0$ :

$$
X(x)=\left.\sum_{m=0}^{\infty} \frac{1}{m !} \overbrace{}^{x m} \odot^{m}{ }^{\times} \nabla^{\prime} X(y)\right|_{y=0}
$$

We then apply (A $5 a$ ) to expand $\overbrace{x}^{\times m}$, and use

$$
\mathfrak{p}\left(\frac{\times(m-2 j)}{\boldsymbol{x}} \stackrel{\times j}{\boldsymbol{A}}\right) \odot^{m} \stackrel{\stackrel{\times m}{\nabla}}{=} \frac{m !}{(m-2 j) ! 2^{j} j !} \stackrel{\times(m-2 j)}{\boldsymbol{x}} \odot^{m-2 j} \stackrel{\times(m-2 j)}{\nabla} \mathrm{D}^{2 j},
$$


(with $D^{2}=A: \nabla \nabla$ as defined in (3.3)), to get

$$
X(x)=\left.\sum_{m=0}^{\infty} \sum_{j=0}^{|m / 2|} \frac{(2 m-4 j+1) ! !}{(2 m-2 j+1) ! !} \frac{1}{(m-2 j) ! 2^{j} j !} \stackrel{x(m-2 j)}{x} \odot^{m-2 j} D^{2 j} \overbrace{\nabla}^{x(m-2 j)} X(y)\right|_{y=0} .
$$

After changing the running index from $m$ to $n=m-2 j$, we find

$$
X(x)=\left.\sum_{n=0}^{\infty} \frac{(2 n+1) ! !}{n !} \overbrace{x}^{\times n} \odot^{n} \sum_{j=0}^{\infty} \frac{1}{(2 n+2 j+1) ! ! 2^{j} j !} \mathrm{D}^{2 j} \stackrel{x^{n}}{\nabla} X(y)\right|_{y-0},
$$

which is equivalent to (A 9).

Finally, we now derive the irreducible expansion of the disturbance velocity for an arbitrary ellipsoid in Stokes flow. The boundary integral equation (1.2) (Ladyzhenskaya 1963, chap. 3), applied to one rigid particle in a flow $u_{0}$, reduces to

$$
u(x)-u_{0}(x)=-\frac{1}{8 \pi \mu} \int_{A} J(x-y) \cdot \sigma(y) \cdot \mathrm{d} A_{y} .
$$

Introduction of the identity (A 9) with $X=\boldsymbol{J}$ into (A 14) yields

$$
\begin{aligned}
& u(x)-u_{0}(x)= \\
& \left.\frac{-1}{8 \pi \mu} \sum_{m=0}^{\infty} \frac{(2 m+1) ! !}{m !} \int_{A} n \cdot \sigma \stackrel{\overbrace{m}^{y}}{y} A_{y} \odot^{m+1}\left[\left(\frac{1}{\mathrm{D}} \frac{\mathrm{d}}{\mathrm{dD}}\right)^{m} \frac{\sinh \mathrm{D}}{\mathrm{D}}\right] \overbrace{\bar{z}}^{\times m} J(x-z)\right|_{z=0},
\end{aligned}
$$

which is identical to (3.2).

\section{Appendix B. Coefficients $\alpha$ and $\gamma$ characterizing the hydrodynamic behaviour of isolated prolate spheroids}

In terms of the eccentricity $e$, which is related to the aspect ratio $r_{p}$ by $e^{2}=1-r_{p}^{-2}$, the coefficients for the self-terms read (Chwang \& Wu 1974, 1975; Kim 1985b):

$$
\begin{aligned}
\alpha_{1}= & e^{2}\left\{-2 e+\left(1+e^{2}\right) \log ((1+e) /(1-e))\right\}^{-1}, \\
\alpha_{2}= & 2 e^{2}\left\{2 e+\left(3 e^{2}-1\right) \log ((1+e) /(1-e))\right\}^{-1}, \\
\gamma= & \left(1-e^{2}\right)\left\{2 e+\left(1-e^{2}\right) \log ((1+e) /(1-e))\right\}^{-1}, \\
\gamma^{\prime}= & \left(2-e^{2}\right)\left\{-2 e+\left(1+e^{2}\right) \log ((1+e) /(1-e))\right\}^{-1}, \\
\gamma_{3}^{\prime}= & \left\{-2 e+\left(1+e^{2}\right) \log ((1+e) /(1-e))\right\}^{-1}, \\
\alpha^{*}= & e^{2} \gamma_{3}^{\prime}\left\{2 e\left(2 e^{2}-1\right)+\left(1-e^{2}\right) \log ((1+e) /(1-e))\right\} \\
& \times\left\{2 e\left(2 e^{2}-3\right)+3\left(1-e^{2}\right) \log ((1+e) /(1-e))\right\}^{-1}, \\
\alpha_{5}= & e^{2}\left\{6 e-\left(3-e^{2}\right) \log ((1+e) /(1-e))\right\}^{-1}, \\
\alpha_{4}= & 2 e^{2}\left(1-e^{2}\right)\left\{\left(2 e\left(3-5 e^{2}\right)-3\left(1-e^{2}\right)^{2} \log ((1+e) /(1-e))\right\}^{-1} .\right.
\end{aligned}
$$

The results for spherical particles can be recovered by noting that

$$
\begin{gathered}
\lim _{e \rightarrow 0} \alpha_{1}=\lim _{e \rightarrow 0} \alpha_{2}=\frac{3}{8} e^{-1}, \\
\lim _{e \rightarrow 0} \gamma=\lim _{e \rightarrow 0} \gamma^{\prime}=\frac{3}{4} e^{-3}, \\
\lim _{e \rightarrow 0} \alpha_{4}=-\frac{5}{8} e^{-3}, \lim _{e \rightarrow 0} \alpha_{5}=-\frac{15}{8} e^{-3}, \quad \lim _{e \rightarrow 0} \alpha^{*}=-\frac{5}{4} e^{-3} .
\end{gathered}
$$




\section{Appendix C. Relations between the mobility tensors of hydrodynamically interacting ellipsoids}

It should not be surprising that a relation linking $\boldsymbol{M}_{U T}$ to $\boldsymbol{M}_{U S}$ exists: both tensors couple the velocity of one particle to the first moment of the force density on another body. Equation (5.1) then shows that enough information is contained in the coupling to the symmetric part of the stress multipole (i.e. the stresslet $S$ ) to deduce the mobility response due to the antisymmetric half (the torque $T$ ). We explore the origin of this relation here.

From the definitions of the torque and stresslet, and of the first multipole $\boldsymbol{P}_{q}^{(1)}$ exerted by a particle $q$, we get (see (A $4 c$ ))

$$
T_{q}=\varepsilon: P_{q}^{(1)} \quad \text { and } \quad S_{q}=\left[\frac{1}{2}(\widehat{\delta} \hat{\delta}+\breve{\delta \delta})-\frac{1}{3} \delta \delta\right]: P_{q}^{(1)}=\varsigma: P_{q}^{(1)}
$$

where we introduced the notations

Inversely,

$$
(\hat{\delta \delta})_{i j k l}=\delta_{i k} \delta_{j l} \text { and }(\widetilde{\delta \delta})_{i j k l}=\delta_{i l} \delta_{j k}
$$

$$
P_{q}^{(1)}=S_{q}-\frac{1}{2} \varepsilon \cdot T_{q}+\frac{1}{3} \int_{A_{q}}\left[y-x_{q}\right] \cdot \sigma \cdot \mathrm{d} A_{y} \delta .
$$

The last term on the right-hand side is inconsequential in incompressible media, and will not be considered further. We now introduce the mobility tensor $M_{01}$ which couples the 0th gradient of the particle velocity (relative to the ambient flow) to the 1st moment of the force density on the particle surface. By definition then, if we ignore the contribution to the translational motion of particle $p$ of all objects other than $q$ and of all stress multipoles other than $\boldsymbol{P}_{q}^{(1)}$, we have

$$
U_{p}-u_{0}=M_{01}^{p q}: P_{q}^{(1)}=M_{01}^{p q}: S_{q}-\frac{1}{2} M_{01}^{p q}: \varepsilon \cdot T_{q} .
$$

We can clearly identify the more familiar tensors $M_{U T}$ and $M_{U S}$ as

$$
M_{U T}^{p q}=-\frac{1}{2} M_{01}^{p q}: \varepsilon \text { and } M_{U S}^{p q}=M_{01}^{p q}: \varsigma \text {. }
$$

Note that $\boldsymbol{M}_{U S}$ is defined as being symmetric and traceless in its last two indices, since it is otherwise indeterminate (Kim \& Mifflin 1985; Brenner 1964a). To convert $\boldsymbol{M}_{\mathbf{0 1}}$ into $M_{U S}$, we thus need to multiply it by $\varsigma$ (see (C 1$)$ ).

We shall derive the relations (5.1) for ellipsoids in what follows, but conjecture that they hold for a wider class of body geometries. Indeed, our proof hinges mostly on the existence of singularity solutions to describe the dynamics of the particles in Stokes flow, and these certainly are not limited to ellipsoids (Chwang \& Wu 1974, 1975). We shall sometimes hint how to generalize our reasoning, but leave the details of a formal theorem up to the interested reader.

We explained in $\S 3$ how to construct the mobility coefficients by combining the disturbance velocity and the Faxén relations for the particles. In the case of ellipsoids, the non-local representation (Kim 1986) clearly shows that each element of the mobility tensor can be written as a linear functional of the Oseen tensor and higher singularities. For instance,

$$
\begin{aligned}
M_{U F}^{p q}= & \frac{1}{32 \pi^{3} \mu c_{E_{p}} c_{E_{p}}^{\prime} c_{E_{q}} c_{E_{q}}^{\prime}} \iint_{E_{p}} \iint_{E_{q}} q_{p}^{-1}(x)\left\{1+\frac{1}{2} a_{3 p}^{2} q_{p}^{2} \nabla^{2}\right\} \\
& q_{q}^{-1}\left(x^{\prime}\right)\left\{1+\frac{1}{2} a_{3 q}^{2} q_{q}^{2} \nabla^{2}\right\} J\left(x-x^{\prime}\right) \mathrm{d} A_{p} \mathrm{~d} A_{q} \\
= & \mathcal{O}_{00}(\mathcal{J})
\end{aligned}
$$


with $x \in E_{p}$ and $x^{\prime} \in E_{q}$. All other symbols have been introduced in $\S 3$, except the operator $\mathcal{O}_{00}$ which is defined, for an arbitrary tensorial function $\boldsymbol{X}$ of the separation vector $\boldsymbol{x}-\boldsymbol{x}^{\prime}$, as

$$
\mathcal{O}_{00}(X)=\frac{1}{32 \pi^{3} \mu c_{E_{p}} c_{E_{p}}^{\prime} c_{E_{q}} c_{E_{q}}^{\prime}} \iint_{E_{p}} \iint_{E_{q}} q_{p}^{-1} q_{q}^{-1}\left\{1+\frac{1}{2}\left(a_{3 p}^{2} q_{p}^{2}+a_{3 q}^{2} q_{q}^{2}\right) \nabla^{2}\right\} X \mathrm{~d} A_{p} \mathrm{~d} A_{q}
$$

In general, it follows from Appendix A (see also Kim \& Arunachalam 1987) that the mobility interaction between the $m$ th gradient of the velocity at the particle locator point and the $\underset{m+n}{n \text {th }}$ irreducible stress multipole is a linear functional of an appropriate transpose of $\overbrace{\boldsymbol{\nabla}}^{\boldsymbol{m}} \mathrm{J}$. In particular, the coupling between the translational velocity $U_{p}-u_{0}\left(x_{p}\right)$ and the first moment of the force density $I_{p}^{(1)}$ involves ${ }^{\mathrm{T}} \nabla \boldsymbol{J}$ only. (This is not necessarily so; counterexamples can be found among the class of bodies with screwsymmetry.) Note also that $I_{q}^{(1)}=P_{q}^{(1)}$ for ellipsoids, so that the equations (C 1)-(C 5) still hold in terms of the irreducible multipole.

One can now write the Oseen tensor $J(r)$ as $\left(\nabla^{2} \delta-\nabla \nabla\right) r$, an identity first used by Beenakker (1986) to facilitate the application of the Ewald summation to hydrodynamic interactions in periodic suspensions. This brings out the symmetry which we exploit to derive the relations (5.1):

$$
\boldsymbol{M}_{01}=\mathcal{O}_{01}\left({ }^{\mathrm{T}} \nabla \boldsymbol{J}\right)=\mathcal{O}_{01}\left(\left(\nabla^{2} \mathrm{~T} \nabla \boldsymbol{\delta}-\nabla \nabla \nabla\right) r\right) .
$$

Since $\mathcal{O}_{01}$ consists of linear non-tensorial operations only (such as integration and differentiation with respect to a coordinate, multiplication by a scalar function of position, etc.), the contractions given by (C 5) commute with the operator and

$$
\begin{aligned}
& \left.M_{U S}=\mathcal{O}_{01}\left({ }^{\mathrm{T}} \nabla J\right): \varsigma\right)=\mathcal{O}_{01}\left(\left(\frac{1}{2} \nabla^{2}\left[\delta \nabla^{\mathrm{T}}+\delta \nabla\right]-\nabla \nabla \nabla\right) r\right), \\
& M_{U T}=-\frac{1}{2} \mathcal{O}_{01}\left(\left({ }^{\mathrm{T}} \nabla J\right): \varepsilon\right)=\frac{1}{2} \mathcal{O}_{01}\left(\left(\nabla^{2} \nabla \cdot \varepsilon\right) r\right) .
\end{aligned}
$$

For $p \neq q$, relation (5.1 $a$ ) now follows as an identity, since

$$
\delta: M_{U S}=\delta: \mathcal{O}_{01}\left(\left(^{T} \nabla J\right): \varsigma\right)=\mathcal{O}_{01}\left(\delta:\left[\left({ }^{T} \nabla J\right): \varsigma\right]\right)=\mathcal{O}_{01}\left(\nabla^{2} \nabla r\right)
$$

so that indeed

$$
\boldsymbol{M}_{U T}^{p q}=\frac{1}{2} \varepsilon \cdot\left(\boldsymbol{\delta}: \boldsymbol{M}_{U S}^{p q}\right)
$$

The other two equations in the set (5.1) can be derived in a similar fashion, but concern couplings between the first gradient of the particle velocity and the first irreducible stress moment. They thus involve a scalar linear functional $\mathcal{O}_{11}$ instead of $\mathcal{O}_{01}$, but the reasoning is the same (Claeys 1991).

For $p=q$, the validity of these relations is most easily demonstrated by checking the identities (5.2). It is then easy to verify $(5.1)$ from the expressions $(3.9 b, c)$ for the mobility tensors $\boldsymbol{M}_{\Omega T}^{p p}, \boldsymbol{M}_{\Omega S}^{p p}$ and $\boldsymbol{M}_{E S}^{p p}$. The relation between $\boldsymbol{M}_{U S}^{p q}$ and $\boldsymbol{M}_{U T}^{p q}$ becomes trivial since both tensors are zero for $p=q$.

The same formalism can also be used to show that $\boldsymbol{M}_{U F}^{p q}$ is symmetric for all pairs of spheroids $p$ and $q$, and that

$$
\left(M_{E S}^{p q}\right)_{i j k l}=\left(M_{E S}^{p q}\right)_{k l i j} .
$$

For $p=q$, this agrees with Lorentz' reciprocal theorem (Brenner 1973; Hinch 1972). The validity of these relations is also easily recognized from (3.11) and the analogous mobility expression for $\boldsymbol{M}_{E S .}^{p q}$. Considered in conjunction with $(5.1 b, c)$, it clearly follows that $\boldsymbol{M}_{\Omega T}^{p q}$ is symmetric and that $\left(\boldsymbol{M}_{\Omega S}^{p q}\right)_{i j k}=\left(\boldsymbol{M}_{E T}^{p q}\right)_{j k i}$ for all $p$ and $q$. 


\section{REFERENCES}

Abramowitz, M. \& Stegun, I. A. 1970 Handbook of Mathematical Functions. Dover.

BARTA, E. \& LIRON, N. 1988 SIAM J. Appl. Maths 48, 1262-1280.

BATCHELOR, G. K. 1970 J. Fluid Mech. 44, 419-440.

BATChelor, G. K. 1971 J. Fluid Mech. 46, 813-829.

Beenakker, C. W. J. 1986 J. Chem. Phys. 85, 1581-1582.

Bitsanis, I., Davis, H. T. \& Tirell, M. 1988 Macromolecules 21, 2824-2835.

Bitsanis, I., Davis, H. T. \& TiRell, M. 1990 Macromolecules 23, 1157-1165.

Bossis, G. \& Brady, J. F. 1984 J. Chem. Phys. 80, 5141-5154.

Bossis, G. \& Brady, J. F. 1989 J. Chem. Phys. 91, 1866-1874.

Brady, J. F. \& Bossis, G. 1988 Ann. Rev. Fluid Mech. 20, 111-157.

Brady, J. F., Phillips, R. J., Lester, J. C. \& Bossis, G. 1988 J. Fluid Mech. 195, 257-280.

Brenner, H. 1963 Chem. Engng Sci. 18, 1-25.

BRENNER, H. 1964 a Chem. Engng Sci. 19, 631-651.

BrenNer, H. $1964 b$ Chem. Engng Sci. 19, 703-727.

Brenner, H. 1966 Chem. Engng Sci. 21, 97-109.

Brenner, H. \& Haber, S. 1963 PhysicoChem. Hydrodyn. 4, 271-278.

Chapman, S. \& Cowling, T. G. 1970 The Mathematical Theory of Non-Uniform Gases. Cambridge University Press.

Chwang, A. T. \& Wu, T. Y. 1974 J. Fluid Mech. 63, 607-622.

Chwang, A. T. Wu, T. Y. 1975 J. Fluid Mech. 67, 787-815.

ClaEYS, I. L. 1988 Experimental and numerical study of molecular rotational diffusion in gel-like media. MS thesis, California Institute of Technology.

ClaeYs, I. L. 1991 Hydrodynamic transport properties of suspensions of non-Brownian prolate spheroids. PhD thesis, California Institute of Technology.

Claeys, I. L. \& Brady, J. F. 1989 PhysicoChem. Hydrodyn. 11, 261-293.

Claeys, I. L. \& Brady, J. F. 1993 a J. Fluid Mech. 251, 443-477.

Claeys, I. L. \& Brady, J. F. $1993 b$ J. Fluid Mech. 251, 479-500.

Cooley, M. D. A. \& O’Neill, M. E. 1969 Proc. Camb. Phil. Soc. 66, 407-415.

Durlofsky, L., Brady, J. F. \& Bossis, G. 1987 J. Fluid Mech. 180, 21-49.

Ermak, D. L. \& McCammon, J. A. 1978 J. Chem. Phys. 69, 1352-1360.

Felderhof, B. U. 1977 Physica 89A, 373-384.

Gluckman, M. J., Pfeffer, R. \& Weinbaum, S. 1971 J. Fluid Mech. 50, 705-740.

HAPPEL, J. \& BrenNer, H. 1973 Low Reynolds Number Hydrodynamics. Martinus Nijhoff.

Hassonjee, Q., Ganatos, P. \& Pfeffer, R. 1988 J. Fluid Mech. 197, 1-37.

HiNCH, E. J. 1972 J. Fluid Mech. 54, 423-425.

Ivanov, Y., Ven, T. G. M. van De \& Mason, S. G. 1982 J. Rheol. 26, 213-230.

Jeffery, G. B. 1922 Proc. R. Soc. Lond. A 102, 161-179.

KIM, S. 1985a Intl J. Multiphase Flow 11, 699-712.

KIM, S. $1985 b$ Intl J. Multiphase Flow 11, 713-719.

KIM, S. 1986 Intl J. Multiphase Flow 12, 469-491.

Kim, S. \& Arunachalam, P. V. 1987 J. Fluid Mech. 178, 535-547.

Kim, S., Fuentes, Y. O. \& Karrila, S. J. 1991 J. Statist. Phys. 62, 1197-1223.

Kim, S. \& Lawrence, C. J. 1988 Chem. Engng Sci. 43, 991-1016.

KIM, S. \& Lu, S. Y. 1987 Intl J. Multiphase Flow 13, 837-844.

Kim, S. \& Mifflin, R. T. 1985 Phys. Fluids 28, 2033-2045.

LADYZHENSKAYA, O. A. 1963 The Mathematical Theory of Viscous Incompressible Flow. Gordon and Breach.

LAMB, H. 1932 Hydrodynamics. Cambridge University Press.

LiaO, W. H. \& KrUeger, D. A. 1980 J. Fluid Mech. 96, 223-241. 
Mazur, P. 1982 Physica 110A, 128-146.

Mazur, P. \& SaARloOs, W. van 1982 Physica 115A, $21-57$.

Mewis, J. \& Metzner, A. B. 1974 J. Fluid Mech. 62, 593-600.

Perry, R. H. \& Green, D. W. 1984 Perry's Chemical Engineer's Handbook. McGraw-Hill.

Phillips, R. J., Brady, J. F. \& Bossis, G. 1988 a Phys. Fluids 31, 3462-3472.

Phillips, R. J., Brady, J. F. \& Bossis, G. 1988 b Phys. Fluids 31, 3473-3479.

Phung, T. \& Brady, J. F. 1992 In Slow Dynamics in Condensed Matter. AIP Conf. Proc., vol. 256 (ed K. Kawasaki, M. Tokuyama \& T. Kawakatsu) pp. 391-400.

Piessens, R., Doncker-Kapenga, E. De, Uberhuber, C. W. \& Kahaner, D. K. 1983 QUADPACK, A Subroutine Package for Automatic Integration. Springer.

Rallison, J. M. 1978 J. Fluid Mech. 88, 529-533.

Rosenberg, J., Denn, M. \& Keunings, R. 1990 J. Non-Newtonian Fluid Mech. 37, 317-345.

Salinas, A. \& Pittman, J. F. T. 1981 Polymer Engng Sci. 21, 23-31.

Shaqfeh, E. S. G. \& Fredrickson, G. H. 1990 Phys. Fluids A 2, 7-24.

SHAQfeH, E. S. G. \& Koch, D. L. 1988 Phys. Fluids 31, 2769-2780.

Stimson, M. \& Jeffer y, G. B. 1926 Proc. R. Soc. Lond. A 111, 110-116.

Weindaum, S., Ganatos, P. \& Yan, Z. Y. 1990 Ann. Rev. Fluid Mech. 22, 275-316.

YoON, B. J. \& Kıм, S. 1990 Intl J. Multiphase Flow 16, 639-649.

Youngren, G. K. \& ACrivos, A. 1975 J. Fluid Mech. 69, 377-403. 